\title{
MODELLING DNA AND RNA SECONDARY STRUCTURES USING MATRIX INSERTION-DELETION SYSTEMS
}

\author{
LAKSHMANAN KUPPUSAMY ${ }^{a}$, ANAND MAHENDRAN ${ }^{b, *}$ \\ ${ }^{a}$ School of Computing Science and Engineering \\ VIT University, Vellore 632014, India \\ e-mail: klakshma@vit.ac.in \\ ${ }^{b}$ Department of Computer Science, College of Computer Science and Information Systems \\ Jazan University, Jazan 45142, Kingdom of Saudi Arabia \\ e-mail: anandmahendran82@gmail.com
}

\begin{abstract}
Insertion and deletion are operations that occur commonly in DNA processing and RNA editing. Since biological macromolecules can be viewed as symbols, gene sequences can be represented as strings and structures can be interpreted as languages. This suggests that the bio-molecular structures that occur at different levels can be theoretically studied by formal languages. In the literature, there is no unique grammar formalism that captures various bio-molecular structures. To overcome this deficiency, in this paper, we introduce a simple grammar model called the matrix insertion-deletion system, and using it we model several bio-molecular structures that occur at the intramolecular, intermolecular and RNA secondary levels.
\end{abstract}

Keywords: bio-molecular structures, insertion-deletion systems, intermolecular, intramolecular, secondary structures.

\section{Introduction}

Natural computing is a field of research that investigates various computing models and computational (algorithms) techniques that are inspired by nature. It is an interdisciplinary area that nudges natural science with computing science, and attempts to understand the world around us in terms of information processing. In the last few decades, natural computing which includes biologically inspired computing, has been pursued with a great deal of interest. This comprises evolutionary computing (Eiben and Smith, 2003), membrane computing (Calude and Paŭn, 2001), genetic algorithms (Goldberg, 1989), DNA computing (Păun et al., 1998), ant colony optimization (Dorigo and Stutzle, 2004) and many other computing models that are inspired from biology and nature. The developments which have taken place in DNA computing inspired the definition and study of new theoretical models in formal language theory, such as sticker systems, splicing systems, Watson-Crick automata, insertion-deletion systems and $P$ systems

\footnotetext{
*Corresponding author
}

(Calude and Paŭn, 2001; Păun et al., 1998; Păun, 2002).

Insertion-deletion systems are introduced to theoretically analyze the insertion and deletion operations that take place in gene sequences. These operations frequently occur in DNA processing and RNA editing. The insertion operation was first studied by Galiukschov (1982). A study of properties of the insertion operation was carried out by Haussler (1982; 1983). Informally, the insertion and deletion operations of an insertion-deletion system are defined as follows: If a string $\beta$ is inserted between two parts $w_{1}$ and $w_{2}$ of a string $w_{1} w_{2}$ to get $w_{1} \beta w_{2}$, we call the operation insertion, whereas if a substring $\alpha$ is deleted from a string $w_{1} \alpha w_{2}$ to get $w_{1} w_{2}$, we call the operation deletion. Consider a production of the form $A \rightarrow c A B$ in a rewriting system. In a derivation step, if there is a presence of non-terminal $A$, then it will be replaced by $c A B$. But in the insertion-deletion system the derivations are obtained either by inserting or by deleting a string. As the system is not exactly based on the rewriting mechanism, it has attracted particular attention in the field of formal language theory.

The biological sequences that occur in DNA, RNA 
and protein molecules can be considered words formed over well-defined chemical alphabets. The DNA molecule consists of sequences that are built of nucleotides, which are in four forms: a (adenine), $t$ (thymine), g(guanine), c(cytosine). The RNA molecule consists of sequences that are built of nucleotides, which are in four forms; $a$, $u$ (uracil), $g, c$. The complementary pair for RNA (DNA) is given as $\bar{a}=u(t), \bar{u}(\bar{t})=a, \bar{g}=c$ and $\bar{c}=g$. Based on the complementary pairs in chemical objects and other biological constraints, sequences form patterns and these patterns are considered structures. These structures play a vital role in governing the functionality and behavior of bio-molecules (Brendel and Busse, 1984; Searls, 1993).

Gene sequence prediction is considered one of the important and fundamental problems in computational biology. Such a sequence prediction problem is dealt with by developing suitable string (pattern) matching algorithms. The above mentioned problem is somewhat akin to analyzing the structural descriptions in computational linguistics. The following example shares a common point between formal languages and molecular strings. Consider the context-free language $L=\left\{w w^{R} \mid w \in\{a, b\}^{*}\right\}$, where $w^{R}$ is the reversal of $w$. For example, if $w$ is aabb, then $w^{R}$ will be bbaa. Consider the gene sequence cggcaacggc. This gene sequence resembles the palindrome (context-free) language $\left\{w w^{R} \mid w \in\{a, u, g, c\}^{*}\right\}$. Also, there exist some relations between bio-molecular sequences and non-context-free natural language constructions such as triple agreements: $\left\{a^{n} b^{n} c^{n} \mid n \geq 1\right\}$, crossed dependencies: $\left\{a^{n} b^{m} c^{n} d^{m} \mid n, m \geq 1\right\}$ and copy language: $\left\{w w \mid w \in\{a, b\}^{*}\right\}$ (Searls, 1992; 1993; 2002). They are discussed in the next paragraph.

We now discuss briefly the bio-molecular structures that are frequently noticed in bio-molecules such as protein, DNA and RNA. Figure 1 shows two popular structures, stem and loop and hairpin, which can be modelled by context-free grammars (Searls, 1988). Figure 2 shows two structures, pseudoknot and attenuator, which are beyond the power of context-free grammars (Searls, 1992). In Figs. 1 and 2, the strings are obtained by reading the symbols as per directed dotted lines. The string cuucaucagaaaaugac represents the stem and loop language (Fig. 1(a)) and the string atcgcgat represents the hairpin language (Fig. 1(b)). The string gcucgcga (Fig. 2(a)) represents the pseudoknot structure and the string gucgacgucgac (refer Fig. 2(b)) represents attenuator structure. Figure 2 shows the coherence between the natural language constructs and the gene sequences. Figure 2(a) represents the pseudoknot structure, which has the crossed dependency pattern, and Figure 2(b) represents the attenuator structure, which has the copy language pattern. The formal language notations for such structures and for a few other structures are discussed in detail in the coming sections. For more details on genome structures, their corresponding languages and gene structure prediction using linguistic methods, we refer to the works of Brendel and Busse (1984), Chiang et al. (2006), Dong and Searls (1994), Durbin et al. (1998), as well as Searls (1988; 1992; 2002).

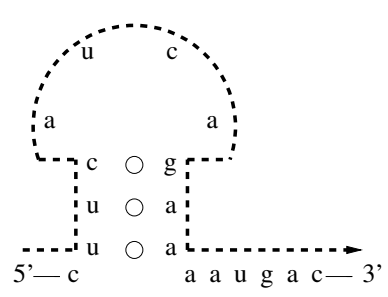

(a)

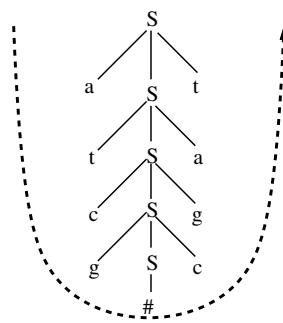

(b)
Fig. 1. Bio-molecular structures: stem and loop (where the $\circ$ denotes the complementary pair) (a), hairpin (where $S$ is a non-terminal for the context-free grammar and \# denotes the empty string) (b).

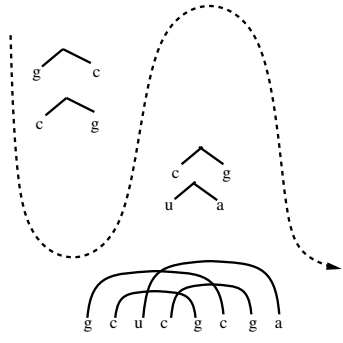

(a)

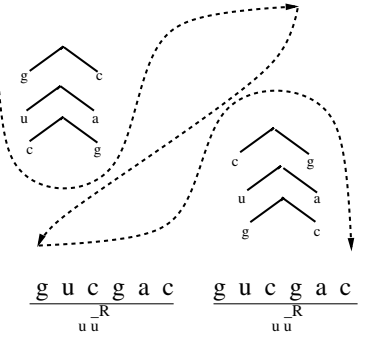

(b)
Fig. 2. Pseudoknot (a), attenuator (b).

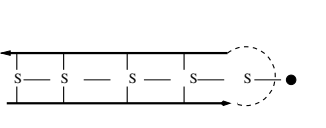

(a)

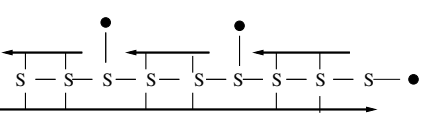

(b)
Fig. 3. Intermolecular structures: double stranded molecule (where $S$ is a non-terminal for the context-free grammar) (a), nick language (where $S$ is a non-terminal for the context-free grammar) (b).

The structures that are formed in RNA are mostly intermolecular. Figure 3 represents some of the intermolecular structures (Searls, 1995): (a) double strand language and (b) nick language, where the cut takes place at arbitrary positions, which is represented by a $\bullet$. The double strand language can be given as $\left\{w \bullet w^{R} \mid w \in\right.$ $\left.\{a, u, g, c\}^{*}\right\}$. In a double stranded molecule, if the cut 
is done at many places, then the molecule is said to be nicked.

The study of linguistic behavior of biological sequences using formal grammars was initiated in the work of Brendel and Busse (1984) as well as Head (1987). Soon after, it was carried out by Searls (1988; 1992; 1993). In this regard, in the last two decades, there have been many attempts made to establish the linguistic behavior of biological sequences by defining new grammar formalisms like cut grammars, ligation grammars (Searls, 1988; 1992; 1993) crossed-interaction grammar (Rivas and Eddy, 2000), simple linear tree adjoining grammars and extended simple linear tree adjoining grammars (Uemura et al., 1999). These are capable of generating some of the biological structures mentioned above.

Like DNA and protein, RNA is also considered one of the important and essential macromolecules that occur in all forms of life. RNA structures are mainly classified as primary, secondary and tertiary structures. The primary structures of a nucleic acid molecule represent the exact sequence of nucleotides that forms the complete molecule. The secondary structures are a two dimensional representation formed by folding back onto itself the base pairing of complementary nucleotides (Watson-Crick pairs). The tertiary structures are 3D structures formed by a single molecule. 3D structures formed by more than one molecule are called quaternary structures. Study of such structures tends to be more complex as it is very difficult to predict the interactions between the molecules. In an RNA secondary structure, the basic

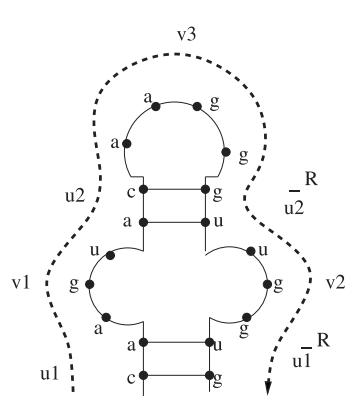

(a)

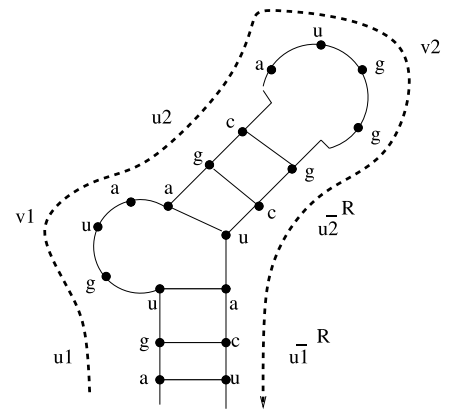

(b)
Fig. 4. RNA secondary structure: internal loop (a), bulge loop (b).

structural motifs can be classified as stem and loop $(\mathrm{H}$ loop) (Fig. 1(a)), internal loop (I-loop) (Fig. 4(a)), bulge loop (B-loop) (Fig. 4(b)) and multi branch loop (Mloop) (Fig. 5(a)). Pseudoknots are also considered to be a structural motif and are formed later in the folding process. Extended pseudoknots (Fig. 5(b)) and kissing hairpin (Fig. 6) are considered to be a common folding motif belonging to the class of pseudoknots.

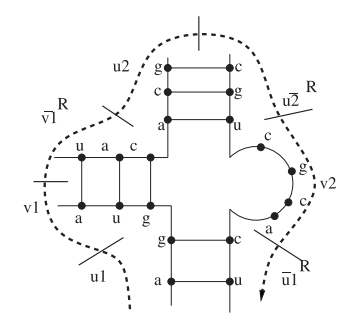

(a)

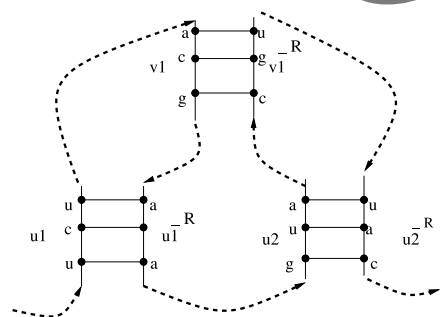

(b)
Fig. 5. RNA secondary structures: multi branch loop (a), extended pseudoknot (b).

To model and predict such structures, many attempts have been made by defining new grammar formalisms like stochastic context-free grammars (Sakakibara et al., 1996), pair hidden Markov models (Sakakibara, 2003) and stochastic multiple context-free grammars (Yuki and Kasami, 2006). In particular, more research work is carried out on RNA pseudoknotted secondary structure prediction. In the work of Theis et al. (2010), prediction of RNA secondary structure is carried out including kissing hairpins. Cai et al. (2003) propose a grammatical approach for stochastic modelling of RNA pesudoknotted structures. In the work of Brown and Wilson (1995), RNA pseudoknot interactions are modelled using the intersection of stochastic context-free grammars. For more details on RNA secondary structures, we refer to the works of Lyngso et al. (1999), Lyngso and Pedersen (2000) or Rivas and Eddy (2000).

Figure 7 shows

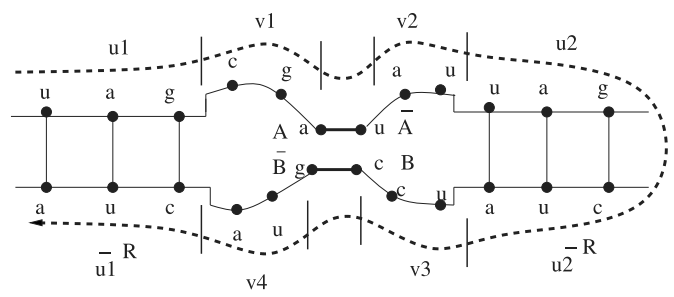

Fig. 6. RNA secondary structure: kissing hairpin.

the simple H-type and recursive pseudoknot structure. Figure 8 presents the three-knot structure. Elements $u_{1}, u_{2}, u_{3}, u_{4}, u_{5}, \bar{u}_{1}^{R}, \bar{u}_{2}^{R}, \bar{u}_{3}^{R}, \bar{u}_{4}^{R}, \bar{u}_{5}^{R}, v, v_{1}, v_{2}, v_{3}$, $v_{4}, A \bar{A}, B \bar{B}$ used in Figs. 4(a), 4(b), 5(a), 5(b), 6, 7(a), 7(b) and 8 are explained in Section 5. Table 1 shows various bio-molecular structures that are commonly noticed in DNA, protein, RNA secondary structures and their corresponding formal grammars which generate the structure.

However, there is no unique grammar model that encapsulates all the above-discussed bio-molecular structures. For example, the double copy language cannot be modelled by a simple linear tree adjoining 


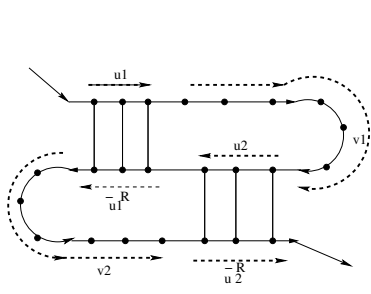

(a)

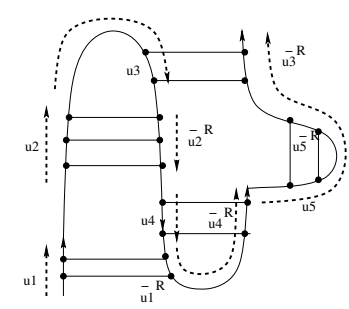

(b)
Fig. 7. RNA secondary structures: simple H-type (a), recursive pseudoknot (b).

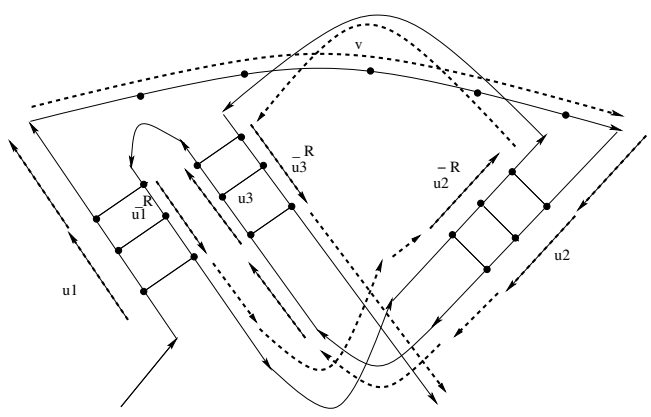

Fig. 8. RNA secondary structure: three-knot structure.

grammar (Uemura et al., 1999). To overcome this failure, we introduced (Kuppusamy et al., 2011a) a simple and powerful grammar model called matrix insertion-deletion systems that captures all the popular and important bio-molecular structures noticed often in bio-molecules. We also modelled the various bio-molecular structures that occur at the intramolecular level such as pseudo knot, hairpin, stem and loop, attenuator (Kuppusamy et al., 2011a). We have modelled the various bio-molecular structures that occur at intermolecular level such as double strand language, nick language, holliday structure, replication fork (Kuppusamy et al., 2011b).

In this paper, we substantially extend our work by introducing many RNA bio-molecular structures (see Section 5) and give a formal language representation for each such structure. Further, we model such structures using the matrix insertion-deletion system. Thus, this paper is an extended journal version of past conference papers (Lakshmanan et al., 2011a; 2011b). Incidentally, in the work of Petre and Verlan (2012), the same matrix insertion-deletion system was discussed and analyzed the computational completeness result for the system. However, the motivation was not from a biological inspiration; it was rather an extension of matrix grammars. In the work of Petre and Verlan (2012), matrix insertion-deletion systems were introduced with the following measures: (i) the maximum number of rules
Table 1. Bio-molecular structure and the corresponding formal grammar.

\begin{tabular}{|c|c|}
\hline $\begin{array}{l}\text { Bio-molecular structure } \\
\text { Figure number(s) }\end{array}$ & Formal grammar \\
\hline $\begin{array}{l}\text { Hairpin } \\
\text { Fig. 1(b) }\end{array}$ & Context free grammar \\
\hline $\begin{array}{l}\text { Stem and loop } \\
\text { Fig. 1(a) }\end{array}$ & Context free grammar \\
\hline $\begin{array}{l}\text { Attenuator } \\
\text { Fig. 2(b) }\end{array}$ & Tree adjoining grammar \\
\hline $\begin{array}{l}\text { Pseudoknot } \\
\text { Fig. 2(a) }\end{array}$ & Tree adjoining grammar \\
\hline $\begin{array}{l}\text { Cloverleaf } \\
\text { Fig. } 9\end{array}$ & Context free grammar \\
\hline $\begin{array}{c}\text { Nick } \\
\text { Fig. 3(b) }\end{array}$ & Cut grammar \\
\hline $\begin{array}{l}\text { Double strand } \\
\text { Fig. 3(a) }\end{array}$ & Ligation grammar \\
\hline $\begin{array}{l}\text { Holliday } \\
\text { Fig. } 10\end{array}$ & Ligation grammar \\
\hline $\begin{array}{l}\text { RNA structures } \\
\text { Figs. } 4(\mathrm{a}), 4(\mathrm{~b}), 5(\mathrm{a}), 5(\mathrm{~b}) \\
\text { 6, 7(a), 7(b), } 8\end{array}$ & $\begin{array}{c}\text { Stochastic CFG } \\
\text { Multiple stochastic CFG } \\
\text { Pair HMM } \\
\text { Stochastic multiple CFG }\end{array}$ \\
\hline
\end{tabular}

in a matrix is denoted by $k$, (ii) the maximal length of the left and right context used in the insertion rules is denoted with $m$ and $m^{\prime}$, respectively, (iii) the maximal length of the left and right context used in deletion rules is denoted with $q$ and $q^{\prime}$, respectively, (iv) the maximal length of the inserted string is denoted with $n$, (v) the maximal length of the deleted string is denoted with $p$.

Based on these measures, the family of languages generated by matrix insertion-deletion systems is denoted by $\operatorname{Mat}_{k} I N S_{n}^{m, m^{\prime}} D E L_{p}^{q, q^{\prime}}$. With these measures, in the work of Petre and Verlan (2012), the computational completeness result (i.e., showing equivalence to recursively enumerable languages) for the matrix insertion-deletion system was proved for the combinations $\mathrm{Mat}_{3} I N S_{1}^{1,0} \mathrm{DEL}_{2}^{0,0}, \quad \mathrm{Mat}_{3} I N S_{1}^{1,0} \mathrm{DEL}_{1}^{1,0}, \mathrm{Mat}_{3}$ $I N S_{1}^{1,0} D E L_{1}^{0,1}$ and $\operatorname{Mat}_{3} I N S_{2}^{0,0} D E L_{1}^{1,0}$. In the same paper, with binary matrices (matrices having two rules) the computational completeness result was proved for the combinations $\mathrm{Mat}_{2} I N S_{2}^{0,0} D E L_{1}^{1,0}$ and $\mathrm{Mat}_{2} I N S_{1}^{1,0} D E L_{2}^{0,0}$. Note that, in these results when no context is considered in deletion rules, the maximal length of the deleted string is 2 . Also, insertion and deletion rules are together used in a matrix. In this paper, we have modelled the bio-molecular structures using the matrix insertion-deletion systems where the length of the deletion string is 1 only. Also, insertion and deletion rules are not used together in a matrix.

This paper is organized as follows. In Section 2, 
we deal with the preliminaries. In Section 3, we briefly introduce matrix insertion-deletion systems. In Section 4, we show that these systems can encompass several essential bio-molecular structures that occur at intramolecular and intermolecular levels in DNA and RNA. In Section 5, we give the language representation for RNA secondary structures and we model them using our grammar model. In Section 6, we conclude the paper with further research direction.

\section{Preliminaries}

We start with recalling some basic notation used in formal language theory. A finite non-empty set $V$ or $\Sigma$ is called an alphabet. $\Sigma_{D N A}$ is a finite non-empty set over the symbols $\{a, t, g, c\} . \Sigma_{R N A}$ is a finite non-empty set over the symbols $\{a, u, g, c\}$. We denote by $V^{*}$ or $\Sigma^{*}$ the free monoid generated by $V$ or $\Sigma$, by $\lambda$ its identity or the empty string, and by $V^{+}$or $\Sigma^{+}$the set $V^{*}-\{\lambda\}$ or $\Sigma^{*}-\{\lambda\}$. The elements of $V^{*}$ or $\Sigma^{*}$ are called words or strings. A language $L$ is defined as $L \subseteq \Sigma^{*}$. Let $w$ be a string, and $|w|_{a}$ denote the number of $a$ in $w$. For more details on formal language theory, we refer to Rozenberg and Salomaa (1997).

Next, we recall the basic definition of insertion-deletion systems. Given an insertion-deletion system $\gamma=(V, T, A, R)$, where $V$ is an alphabet (set of non-terminal and terminal symbols), $T \subseteq V$ (set of terminal symbols), $A$ is a finite language over $V, R$ is a set of finite triples of the form $(u, \alpha / \beta, v)$, where $(u, v) \in V^{*} \times V^{*},(\alpha, \beta) \in\left(V^{+} \times\{\lambda\}\right) \cup\left(\{\lambda\} \times V^{+}\right)$. The pair $(u, v)$ is called contexts, which will be used in deletion/insertion rules. The insertion rule is of the form $(u, \lambda / \beta, v)$, which means that $\beta$ is inserted between $u$ and $v$. The deletion rule is of the form $(u, \alpha / \lambda, v)$, which means that $\alpha$ is deleted between $u$ and $v$. In other words, $(u, \lambda / \beta, v)$ corresponds to the rewriting rule $u v \rightarrow u \beta v$, and $(u, \alpha / \lambda, v)$ corresponds to the rewriting rule $u \alpha v \rightarrow u v$.

Consequently, for $x, y \in V^{*}$ we can write $x \Longrightarrow^{*} y$, if $y$ can be obtained from $x$ by using either an insertion rule or a deletion rule which is given as follows (the down arrow $\downarrow$ indicates the position where the string is inserted, the down arrow $\Downarrow$ indicates the position where the string is deleted and the underlined string indicates the string inserted):

(i) $x=x_{1} u^{\downarrow} v x_{2}, y=x_{1} u \beta v x_{2}$, for some $x_{1}, x_{2} \in V^{*}$ and $(u, \lambda / \beta, v) \in R$.

(ii) $x=x_{1} u \alpha v x_{2}, y=x_{1} u^{\Downarrow} v x_{2}$, for some $x_{1}, x_{2} \in V^{*}$ and $(u, \alpha / \lambda, v) \in R$.

The language generated by $\gamma$ is defined by

$$
L(\gamma)=\left\{w \in T^{*} \mid x \Longrightarrow^{*} w, \text { for some } x \in A\right\},
$$

where $\Longrightarrow *$ is the reflexive and transitive closure of the relation $\Longrightarrow$.

Next, we discuss matrix grammar. A matrix grammar is an ordered quadruple $G=(N, T, S, M)$ where $N$ is a set of non-terminals, $T$ is a set of terminals, $S \in N$ is the start symbol and $M$ is a finite set of nonempty sequences whose elements are ordered pairs $(P, Q)$. The pairs are referred to as productions and written in the form $P \rightarrow Q$. The sequences are referred to as matrices and written as $m=\left[P_{1} \rightarrow Q_{1}, \ldots, P_{r} \rightarrow\right.$ $\left.Q_{r}\right], r \geq 1$. For a matrix grammar $G$, the relation $\Longrightarrow$ on the set $V^{*}$ is defined as follows. For any $P, Q \in V^{*}$, $P \Longrightarrow Q$ holds if and only if there exist an integer $r \geq 1$ and words $\beta_{1}, \ldots, \beta_{r+1}, P_{1}, \ldots, P_{r}, Q_{1}, \ldots, Q_{r}$, $R_{1}, \ldots, R_{r}, R^{1}, \ldots, R^{r}$ over $V$ such that (i) $\alpha_{i}=P$ and $\alpha_{r+1}=Q$, (ii) $m$ is one of the matrices of $G$, (iii) $\alpha_{i}=R_{i} P_{i} R^{i}$ and $\alpha_{i+1}=R_{i} Q_{i} R^{i}$. If the above conditions are satisfied, it is also said that $P \Longrightarrow Q$ holds with specifications $\left(m, R_{1}\right)$. The reflexive and transitive closure of $\Longrightarrow$ is denoted by $\Longrightarrow$. The above matrix grammar is without appearance checking. The language generated by the matrix grammar is defined by $L(G)=$ $\left\{w \in T^{*} \mid S \Longrightarrow \Longrightarrow^{*} w\right\}$. A matrix grammar with appearance checking is defined as $G=(N, T, S, M, F)$, where $F$ is a set of occurrences of rules in the matrices of $M$. While deriving, a rule may be exempted to apply if the rule is in $F$. The language generated by the matrix grammar with appearance checking is defined as $L_{a c}(G, F)=\left\{w \in T^{*} \mid S \Longrightarrow{ }^{*} w\right\}$. For more details on matrix grammars, we refer to the work of Rozenberg and Salomaa (1997).

Next, we discuss cut grammars (Searls, 1995) designed specifically for modelling intermolecular structures. A cut grammar $G=(N, T, S, P)$ where $N$ is a finite set of non-terminals, $T$ is a finite set of terminals, $S$ is a start symbol and $P$ is a finite set of productions in $(N \cup T)^{*} N(N \cup T)^{*} \times(N \cup T \cup\{\bullet\})^{*}$ where • is a new symbol called cut symbol not in $N$ or $T$. The language generated by the cut grammar is defined as $L(G)=\left\{w \in(T \cup \bullet)^{*} \mid S \Longrightarrow{ }^{*} w\right\}$.

Given any string $w=w_{1} \bullet w_{2} \bullet \ldots \bullet w_{n}$ where $w_{i} \in T^{*}$ for $1 \leq i \leq n$, the cut function is given as $\widehat{w}=\left\{w_{1}, w_{2}, \ldots, w_{n}\right\}$ and the uncut function is given as $\widetilde{w}=w_{1} w_{2} \ldots w_{n}$. For a given cut grammar $G$ and start symbol $S$, the cut language is defined as $\widehat{L}(G)=\{\widehat{w} \in$ $\left.2^{T^{*}} \mid S \Longrightarrow^{*} w\right\}$ and the uncut language is defined as $\widetilde{L}(G)=\left\{\widetilde{w} \in T^{*} \mid S \Longrightarrow{ }^{*} w\right\}$. With cut grammars, the structures double strand language, nick language, holliday structure are represented. For more details on cut grammars, we refer to the work of Searls (1995).

\section{Matrix insertion-deletion systems}

In this section, we explain the grammar model matrix insertion-deletion systems. A matrix insertion-deletion 
system is a construct $\Upsilon=(V, T, A, R)$, where $V$ is an alphabet, $T \subseteq V, A$ is a finite language over $V, R$ is a set of finite triples in the matrix format $\left[\left(u_{1}, \alpha_{1} / \beta_{1}, v_{1}\right), \ldots,\left(u_{n}, \alpha_{n} / \beta_{n}, v_{n}\right)\right]$, where $\left(u_{k}, v_{k}\right) \in$ $V^{*} \times V^{*}$, and $\left(\alpha_{k}, \beta_{k}\right) \in\left(V^{+} \times\{\lambda\}\right) \cup\left(\{\lambda\} \times V^{+}\right)$, with $\left(u_{k}, \alpha_{k} / \beta_{k}, v_{k}\right) \in R_{I_{i}} \cup R_{D_{j}} \cup R_{I_{i} / D_{j}}$, for $1 \leq i \leq m$, $1 \leq j \leq m, 1 \leq k \leq n$, where $m$ is the number of rules in the matrix format in $R$. Here $R_{I_{i}}$ denotes the matrix which consists of only insertion rules, $R_{D_{j}}$ denotes the matrix which consists of only deletion rules and $R_{I_{i} / D_{j}}$ denotes the matrix which consists of both insertion and deletion rules.

Consequently, for $x, y \in V^{*}$ we can write $x \Longrightarrow$ $x^{\prime} \Longrightarrow x^{\prime \prime} \Longrightarrow \ldots \Longrightarrow y$, if $y$ can be obtained from $x$ by using a matrix consisting of insertion rules $\left(R_{I_{i}}\right)$, or deletion rules $\left(R_{D_{j}}\right)$ or insertion and deletion rules $\left(R_{I_{i} / D_{j}}\right)$. In a derivation step the rules in a matrix are applied sequentially one after the other in order, and no rule is in appearance checking (note that the rules in a matrix are not applied in parallel). The language generated by $\Upsilon$ is defined by

$$
\begin{gathered}
L(\Upsilon)=\left\{w \in T^{*} \mid x \Longrightarrow_{R_{\chi}}^{*} w,\right. \text { for some } \\
\left.x \in A, \chi \in\left\{I_{i}, D_{j}, I_{i} / D_{j}\right\}\right\},
\end{gathered}
$$

where $R_{\chi}$ denotes the matrix rules from an insertion matrix or a deletion matrix or a combination of both the rules. $\Longrightarrow^{*}$ is the reflexive and transitive closure of the relation $\Longrightarrow$. Note that the string $w$ is collected after applying all the rules in a matrix and also $w \in T^{*}$.

\section{Modelling bio-molecular structures}

In this section, we show that matrix insertion-deletion systems can capture the commonly noticed biological structures that are discussed earlier in the paper. In most of the following derivations, in each derivation step, we directly write the resultant string obtained by applying all the rules in a matrix. In all the lemmas, we adopt the method of proof by construction in modelling the bio-molecular structures using matrix insertion-deletion systems. In the derivation step, the rule at the suffix of $\Longrightarrow$ denotes the corresponding matrix rule applied. From the formal language theory perspective, since structures can be viewed as languages, in many places we refer to be structure as language.

\subsection{Representation of intramolecular structures.} In this section, we model some of the bio-molecular structures that occur at the intramolecular level.

Lemma 1. The pseudoknot structure language (see Fig. 2(a)) $L_{p s}=\left\{u v \bar{u}^{R} \bar{v}^{R} \mid u, v \in \Sigma_{D N A}^{*}\right\}$ can be generated by a matrix insertion-deletion system.
Proof. The language $L_{p s}$ can be generated by the matrix insertion-deletion system $\Upsilon_{p s}=$ $\left(\left\{b, \bar{b}, \uparrow_{1}, \dagger_{2}, \dagger_{3}, \dagger_{4}\right\},\{b\right.$,

$\left.\bar{b}\},\left\{\lambda, \dagger_{1} \dagger_{2} \dagger_{3} \dagger_{4}\right\}, R\right)$, where $b \in\{a, t, g, c\}, \bar{b}$ is complement of $b$ and $R$ is given as follows:

$$
\begin{aligned}
R_{I_{1}} & =\left[\left(\lambda, \lambda / b, \dagger_{1}\right),\left(\lambda, \lambda / \bar{b}, \dagger_{3}\right)\right], \\
R_{I_{2}} & =\left[\left(\lambda, \lambda / b, \dagger_{2}\right),\left(\lambda, \lambda / \bar{b}, \dagger_{4}\right)\right], \\
R_{D_{1}} & =\left[\left(\lambda, \dagger_{1} / \lambda, \lambda\right),\left(\lambda, \dagger_{3} / \lambda, \lambda\right)\right], \\
R_{D_{2}} & =\left[\left(\lambda, \dagger_{2} / \lambda, \lambda\right),\left(\lambda, \dagger_{4} / \lambda, \lambda\right)\right] .
\end{aligned}
$$

The $\Upsilon_{p s}$ generates only the language $L_{p s}$. The idea behind the construction of the system is given as follows. $\dagger_{1}, \dagger_{2}, \dagger_{3}, \dagger_{4}$ are used as markers. Whenever a $b$ is adjoined to the left of $\dagger_{1}$, its corresponding complementary $\bar{b}$ should be adjoined to the left of $\dagger_{3}$ using the rule $R_{I_{1}}$. So, $\dagger_{1}$ and $\dagger_{3}$ are used to control the $u \bar{u}^{R}$ part of the language. Similarly, whenever a $b$ is adjoined to the left of $\dagger_{2}$, its corresponding complementary $\bar{b}$ should be adjoined to the left of $\dagger_{4}$ using the rule $R_{I_{2}}$. So, $\dagger_{2}$ and $\dagger_{4}$ are used to control the $v \bar{v}^{R}$ part of the language. When the rule $R_{D_{1}}$ is used first, then system $\Upsilon_{p s}$ generates only $v \bar{v}^{R}$ part of the language. When the rule $R_{D_{2}}$ is used first, then the system $\Upsilon_{p s}$ generates only the $u \bar{u}^{R}$ part of the language. We present a sample derivation for a better understanding (the rule at the suffix of the derivation symbol $\Longrightarrow$ denotes whether an insertion rule or a deletion rule is applied),

$$
\begin{aligned}
& \downarrow_{\dagger_{1}} \dagger_{2}^{\downarrow} \dagger_{3} \dagger_{4} \Longrightarrow_{R_{I_{1}}} \underline{a} \dagger_{1}^{\downarrow} \dagger_{2} \underline{t} \dagger_{3}^{\downarrow} \dagger_{4} \Longrightarrow_{R_{I_{2}}} \\
& a \dagger_{1} \underline{g}^{\downarrow} \dagger_{2} t \dagger_{3} \underline{c}^{\downarrow} \dagger_{4} \Longrightarrow_{R_{I_{2}}} a \dagger_{1} g \underline{a} \dagger_{2} t \dagger_{3} c \underline{t} \dagger_{4} \\
& \Longrightarrow R_{D_{1}} a^{\Downarrow} g a \dagger_{2} t^{\Downarrow} c t \dagger_{4} \Longrightarrow_{R_{D_{2}}} a g a^{\Downarrow} t c t^{\Downarrow} .
\end{aligned}
$$

From Fig. 2(b), the attenuator language can be given as $L_{a n}=\left\{u \bar{u}^{R} u \bar{u}^{R} \mid u \in \Sigma_{D N A}^{*}\right\}$.

Lemma 2. The attenuator language $L_{a n}$ (see Fig. 2(b)) can be generated by a matrix insertion-deletion system.

Proof. The language $L_{a n}$ can be generated by the matrix insertion-deletion system

$$
\Upsilon_{a n}=\left(\left\{a, t, g, c, \dagger_{1}, \dagger_{2}\right\},\{a, t, g, c\},\left\{\lambda, \dagger_{1} \dagger_{2}\right\}, R\right),
$$

where $R$ is given as follows:

$$
\begin{aligned}
R_{I_{1}}= & {\left[\left(\lambda, \lambda / a, \dagger_{1}\right),\left(\dagger_{1}, \lambda / t, \lambda\right),\left(\lambda, \lambda / a, \dagger_{2}\right),\right.} \\
& \left.\left(\dagger_{2}, \lambda / t, \lambda\right)\right], \\
R_{I_{2}}= & {\left[\left(\lambda, \lambda / t, \dagger_{1}\right),\left(\dagger_{1}, \lambda / a, \lambda\right),\left(\lambda, \lambda / t, \dagger_{2}\right),\right.} \\
& \left.\left(\dagger_{2}, \lambda / a, \lambda\right)\right], \\
R_{I_{3}}= & {\left[\left(\lambda, \lambda / c, \dagger_{1}\right),\left(\dagger_{1}, \lambda / g, \lambda\right),\left(\lambda, \lambda / c, \dagger_{2}\right),\right.} \\
& \left.\left(\dagger_{2}, \lambda / g, \lambda\right)\right], \\
R_{I_{4}}= & {\left[\left(\lambda, \lambda / g, \dagger_{1}\right),\left(\dagger_{1}, \lambda / c, \lambda\right),\left(\lambda, \lambda / g, \dagger_{2}\right),\right.} \\
& \left.\left(\dagger_{2}, \lambda / c, \lambda\right)\right], \\
R_{D_{1}}= & {\left[\left(\lambda, \dagger_{1} / \lambda, \lambda\right),\left(\lambda, \dagger_{2} / \lambda, \lambda\right)\right] . }
\end{aligned}
$$


Here $\Upsilon_{a n}$ generates only the language $L_{a n}$. Marker $\dagger_{1}$ is used to control the first part of the language $\left(u \bar{u}^{R}\right)$ and marker $\dagger_{2}$ is used to control the second part of the language $\left(u \bar{u}^{R}\right)$. Whenever a $b$ and its corresponding complementary $\bar{b}$ are adjoined by using the $\dagger_{1}$, simultaneously by using the $\dagger_{2}$, the same $b$ and its complementary $\bar{b}$ are adjoined. As the rule $R_{I_{1}}$ uses both the markers $\dagger_{1}$ and $\dagger_{2}$, synchronization is easily maintained. A similar procedure holds for the remaining rules $R_{I_{2}}, R_{I_{3}}$ and $R_{I_{4}}$, e.g.,

$$
\begin{aligned}
& \downarrow \dagger_{1}^{\downarrow} \dagger_{2}^{\downarrow} \Longrightarrow R_{I_{1}} \underline{a}^{\downarrow} \dagger_{1}^{\downarrow} \underline{t} \underline{a}^{\downarrow} \dagger_{2}^{\downarrow} \underline{t} \Longrightarrow R_{I_{2}} \\
& a \underline{t}^{\downarrow} \dagger_{1}^{\downarrow} \underline{a} t a \underline{t}^{\downarrow} \dagger_{2}^{\downarrow} \underline{a} t \Longrightarrow_{R_{I_{3}}} a t \underline{c}^{\downarrow} \dagger_{1}^{\downarrow} \underline{g a t a t}^{\downarrow} \dagger_{2}^{\downarrow} \underline{g} a t \\
& \Longrightarrow R_{I_{4}} \text { atcg } \dagger_{1} \underline{\text { cgatatcg }} \dagger_{2} \underline{\text { cgat }} \Longrightarrow R_{D_{1}} \\
& \text { atcg }{ }^{\Downarrow} \text { cgatatcg }{ }^{\Downarrow} \text { cgat. }
\end{aligned}
$$

Lemma 3. The hairpin language (see Fig. $1(b)$ ) $L_{h p}=$ $\left\{w \bar{w}^{R} \mid w \in \Sigma_{D N A}^{*}\right\}$ can be generated by a matrix insertion-deletion system.

Proof. The hairpin language $L_{h p}$ can be generated by the matrix insertion-deletion system

$$
\Upsilon_{h p}=(\{b, \bar{b}, \dagger\},\{b \bar{b}\},\{\lambda, \dagger\}, R),
$$

where $b \in\{a, t, g, c\}, \bar{b}$ is complement of $b$ and $R$ is given as follows:

$$
\begin{aligned}
R_{I_{1}} & =[(\lambda, \lambda / b, \dagger),(\dagger, \lambda / \bar{b}, \lambda), \\
R_{D_{1}} & =[(\lambda, \dagger / \lambda, \lambda)] .
\end{aligned}
$$

We present a sample derivation which itself suffices to see that $L\left(\Upsilon_{h p}\right)=L_{h p}$,

$$
\begin{aligned}
& \downarrow^{\downarrow} \Longrightarrow R_{I_{1}} \underline{t}^{\downarrow} \dagger^{\downarrow} \underline{a} \Longrightarrow R_{I_{1}} \underline{t g}^{\downarrow} \dagger^{\downarrow} \underline{c} a \Longrightarrow_{R_{I_{1}}} \\
& t g \underline{c}^{\downarrow} \dagger^{\downarrow} \underline{g} c a \Longrightarrow R_{I_{1}} \operatorname{tg} c \underline{g}^{\downarrow} \dagger^{\downarrow} c \underline{g} c a \Longrightarrow R_{D_{1}} \\
& \operatorname{tgc} \underline{g}^{\Downarrow} \underline{c} g c a .
\end{aligned}
$$

Lemma 4. The stem and loop language (see Fig. 1(a)) $L_{s l}=\left\{u v \bar{u}^{R} \mid u, v \in \Sigma_{D N A}^{*}\right\}$ can be generated by a matrix insertion-deletion system.

Proof. The stem and loop language $L_{s l}$ can be generated by the matrix insertion-deletion system $\Upsilon_{s l}=$ $\left(\left\{b, \bar{b}, \dagger_{1}, \dagger_{2}, \dagger_{3}\right\},\{b, \bar{b}\},\left\{\lambda, \dagger_{1} \dagger_{3} \dagger_{2}\right\}, R\right)$, where $b \in$ $\{a, t, g, c\}, \bar{b}$ is the complement of $b$ and $R$ is given as follows:

$$
\begin{aligned}
R_{I_{1}} & =\left[\left(\lambda, \lambda / b, \dagger_{1}\right),\left(\dagger_{2}, \lambda / \bar{b}, \lambda\right)\right], \\
R_{I_{2}} & =\left[\left(\lambda, \lambda / b, \dagger_{3}\right)\right], \\
R_{D_{1}} & =\left[\left(\lambda, \dagger_{1} / \lambda, \lambda\right),\left(\lambda, \dagger_{2} / \lambda, \lambda\right)\right], \\
R_{D_{2}} & =\left[\left(\lambda, \dagger_{3} / \lambda, \lambda\right)\right] .
\end{aligned}
$$

A sample derivation is given follows:

$$
\begin{aligned}
& \downarrow^{\downarrow} \dagger_{1} \dagger_{3} \dagger_{2}^{\downarrow} \\
& \Longrightarrow_{R_{I_{1}}} \underline{c} \dagger_{1}^{\downarrow} \dagger_{3} \dagger_{2} \underline{g} \Longrightarrow_{R_{I_{2}}} \\
& c \dagger_{1} \underline{t}^{\downarrow} \dagger_{3} \dagger_{2} g \Longrightarrow{ }_{R_{I_{2}}} c \dagger_{1} t \underline{c} \dagger_{3} \dagger_{2} g \\
& \Longrightarrow_{R_{D_{1}}} c^{\Downarrow} t c \dagger_{3}^{\Downarrow} g \Longrightarrow R_{D_{2}} c t c^{\Downarrow} g .
\end{aligned}
$$

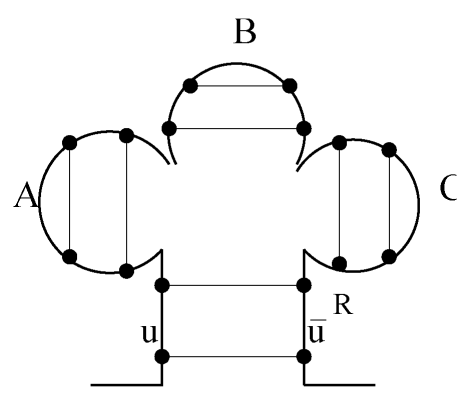

Fig. 9. Cloverleaf representation (where $A=v_{1} \bar{v}_{1}^{R}, B=$ $\left.v_{2} \bar{v}_{2}^{R}, C=v_{3} \bar{v}_{3}^{R}\right)$.

Lemma 5. The cloverleaf language (see Fig. 9, for $n=3$ ) (Searls, 1988; 1992)

$$
\begin{array}{r}
L_{c l}=\left\{u v_{1} \bar{v}_{1}^{R} v_{2} \bar{v}_{2}^{R} \ldots v_{n} \bar{v}_{n}^{R} \bar{u}^{R} \mid u, v_{1}, v_{2}, \ldots,\right. \\
\left.v_{n} \in \Sigma_{D N A}^{*}, n \geq 0\right\}
\end{array}
$$

can be generated by a matrix insertion-deletion system.

Proof. The cloverleaf language $L_{c l}$ (for $n=3$ ) can be generated by the matrix insertion-deletion system $\Upsilon_{c l}=$ $\left(\left\{b, \bar{b}, \dagger_{1}, \dagger_{2}, \dagger_{3}, \dagger_{4}, \dagger_{5}\right\},\{b, \bar{b}\},\left\{\lambda, \dagger_{1} \dagger_{2}, \dagger_{3} \dagger_{4} \dagger_{5}, \dagger_{1} \dagger_{3} \dagger_{4} \dagger_{5}\right.\right.$ $\left.\left.\dagger_{2}\right\}, R\right)$, where $b \in\{a, t, g, c\}, \bar{b}$ is a complement of $b$ and $R$ is given as follows:

$$
\begin{aligned}
R_{I_{1}} & =\left[\left(\lambda, \lambda / b, \dagger_{1}\right),\left(\dagger_{2}, \lambda / \bar{b}, \lambda\right)\right], \\
R_{I_{2}} & =\left[\left(\lambda, \lambda / b, \dagger_{3}\right),\left(\dagger_{3}, \lambda / \bar{b}, \lambda\right)\right], \\
R_{I_{3}} & =\left[\left(\lambda, \lambda / b, \dagger_{4}\right),\left(\dagger_{4}, \lambda / \bar{b}, \lambda\right)\right], \\
R_{I_{4}} & =\left[\left(\lambda, \lambda / b, \dagger_{5}\right),\left(\dagger_{5}, \lambda / \bar{b}, \lambda\right)\right], \\
R_{D_{1}} & =\left[\left(\lambda, \dagger_{1} / \lambda, \lambda\right),\left(\lambda, \dagger_{2} / \lambda, \lambda\right)\right], \\
R_{D_{2}} & =\left[\left(\lambda, \dagger_{3} / \lambda, \lambda\right)\right], \\
R_{D_{3}} & =\left[\left(\lambda, \dagger_{4} / \lambda, \lambda\right)\right], \\
R_{D_{4}} & =\left[\left(\lambda, \dagger_{5} / \lambda, \lambda\right)\right] .
\end{aligned}
$$


A sample derivation is given as follows:

$$
\begin{aligned}
& \downarrow \dagger_{1} \dagger_{3} \dagger_{4} \dagger_{5} \dagger_{2}^{\downarrow} \Longrightarrow R_{I_{1}} \underline{c}^{\downarrow} \dagger_{1} \dagger_{3} \dagger_{4} \dagger_{5} \dagger_{2}^{\downarrow} \underline{g} \\
& \Longrightarrow R_{I_{1}} c \underline{g} \dagger_{1}^{\downarrow} \dagger_{3}^{\downarrow} \dagger_{4} \dagger_{5} \dagger_{2} \underline{c} g t \\
& \Longrightarrow R_{I_{2}} a c g \dagger_{1} \underline{t} \dagger_{3} \underline{a}^{\downarrow} \dagger_{4}^{\downarrow} \dagger_{5} \dagger_{2} c g \\
& \Longrightarrow R_{I_{3}} c g \dagger_{1} t \dagger_{3} a \underline{c} \dagger_{4} \underline{g}^{\downarrow} \dagger_{5} \dagger_{2} c g \\
& \Longrightarrow R_{I_{4}} c g \dagger_{1} t \dagger_{3} a c \dagger_{4} g \underline{a} \dagger_{5} \underline{t} \dagger_{2} c g \\
& \Longrightarrow R_{D_{1}} c g t^{\Downarrow} \dagger_{3} a c \dagger_{4} g a \dagger_{5} t^{\Downarrow} c g \\
& \Longrightarrow R_{D_{2}} c g t^{\Downarrow} a c \dagger_{4} g a \dagger_{5} t c g \\
& \Longrightarrow R_{D_{3}} c g t a c^{\Downarrow} g a \dagger_{5} t c g \\
& \Longrightarrow R_{D_{4}} c g t a c g a^{\Downarrow} t c g .
\end{aligned}
$$

Using five markers, $\dagger_{1}, \dagger_{2}, \dagger_{3}, \dagger_{4}, \dagger_{5}$ the system $\Upsilon_{c l}$ generates the cloverleaf language $L_{c l}$ for $n=3$. By introducing more markers, the system $\Upsilon_{c l}$ can generate a cloverleaf language for an arbitrary value of $n$.

4.2. Representation of intermolecular structures. In this section, we model some of the bio-molecular structures that occur at intermolecular level.

Lemma 6. The double strand language

$$
L_{d s}=\left\{u \bullet \bar{u}^{R} \mid u \in \Sigma_{D N A}^{*}\right\}
$$

can be modelled by a matrix insertion-deletion system.

Proof. $\quad$ The double strand language (see Fig. 3(a)) $L_{d s}$ can be be modelled by a matrix insertion-deletion system $\Upsilon_{d s}=(\{b, \bar{b}, \bullet\},\{b, \bar{b}, \bullet\},\{\bullet\}, R)$ where $b \in$ $\{a, t, g, c\}, \bar{b}$ is complement of $b$ and $R$ is given as $R_{I_{1}}=$ $[(\lambda, \lambda / b, \bullet),(\bullet, \lambda / \bar{b}, \lambda)]$. We present a sample derivation which itself is sufficient to see that $L\left(\Upsilon_{d s}\right)=L_{d s}$,

$$
\begin{aligned}
& \downarrow_{\bullet \downarrow} \Longrightarrow R_{I_{1}} \underline{a}^{\downarrow} \bullet \downarrow-\underline{t} \Longrightarrow R_{I_{1}} a \underline{g}^{\downarrow} \bullet^{\downarrow} \underline{c} t \Longrightarrow R_{I_{1}} \\
& a g \underline{a}^{\downarrow} \bullet{ }^{\downarrow} \underline{t} c t \Longrightarrow R_{I_{1}} a g a \underline{c}^{\downarrow} \bullet \downarrow \text { gtct. }
\end{aligned}
$$

From Fig. 3(b) the nick language can be informally described as $L_{n l}=\left\{w_{1} \bullet w_{2} \mid \widetilde{w}_{2}=\bar{w}_{1}^{R}\right\}$, where $w_{1} \in$ $\Sigma^{*}$ and $w_{2} \in(\Sigma \cup\{\bullet\})^{*}$ (i.e., $w_{2}$ is a string which contains a number of $\bullet$ ).

Lemma 7. The nick language $L_{n l}$ can be generated by matrix insertion-deletion system.

Proof. The nick language (see Fig. 3(b)) $L_{n l}$ can be generated by the cut grammar $G_{n l}=S \rightarrow b S \bar{b} \mid$ $S \bullet \mid \bullet$ for each $b \in \Sigma_{D N A}$. The grammar $G_{n l}$ can be modelled by the matrix insertion-deletion system
$\Upsilon_{n l}=(\{b, \bar{b}, \dagger, \bullet\},\{b, \bar{b}, \bullet\},\{b \dagger \bar{b}, \dagger \bullet, \bullet\}, R)$ where $b \in$ $\{a, t, g, c\}, \bar{b}$ is a complement of $b$ and $R$ is given as

$$
\begin{aligned}
R_{I_{1}} & =[(\lambda, \lambda / b, \dagger),(\dagger, \lambda / \bar{b}, \lambda)], \\
R_{I_{2}} & =[(\dagger, \lambda / \bullet, \lambda)], \\
R_{D_{1}} & =[(\lambda, \dagger / \lambda, \lambda)] .
\end{aligned}
$$

A sample derivation is given as follows:

$$
\begin{aligned}
& a^{\downarrow} \dagger^{\downarrow} t \Longrightarrow R_{I_{1}} a \underline{t}^{\downarrow} \dagger^{\downarrow} \underline{a} t \Longrightarrow R_{I_{1}} a t \underline{g} \dagger^{\downarrow} \underline{c} a t \Longrightarrow_{R_{I_{2}}} \\
& a t g^{\downarrow} \dagger^{\downarrow} \underline{\bullet} a t \Longrightarrow{ }_{R_{I_{1}}} \text { atg } \underline{a} \dagger^{\downarrow} \underline{t} \bullet c a t \Longrightarrow_{R_{I_{2}}} \\
& \text { atga } \dagger \underline{\bullet} \bullet c a t \Longrightarrow{ }_{R_{D_{1}}} \text { atga }{ }^{\Downarrow} \bullet t \bullet c a t .
\end{aligned}
$$

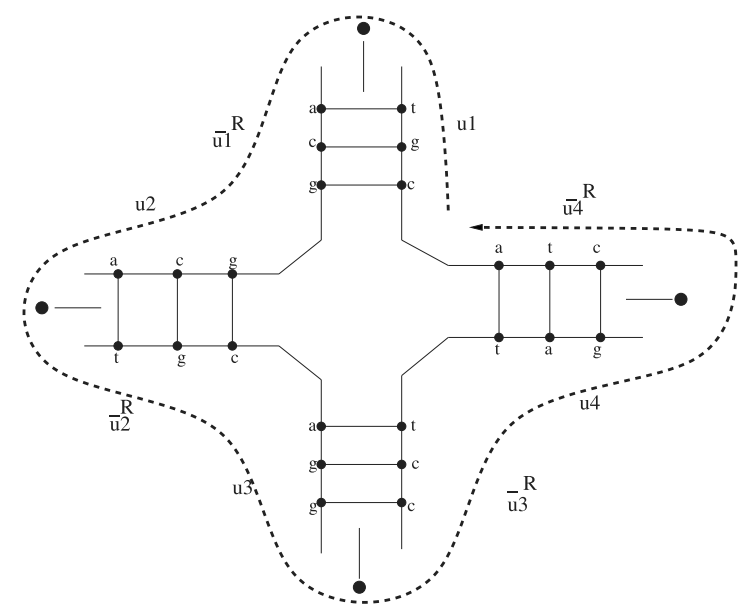

Fig. 10. Holliday structure.

Lemma 8. The holliday structure (see Fig. 10)

$$
\begin{array}{r}
L_{h s}=\left\{u_{1} \bullet{\overline{u_{1}}}^{R} u_{2} \bullet{\overline{u_{2}}}^{R} u_{3} \bullet{\overline{u_{3}}}^{R} u_{4} \bullet{\overline{u_{4}}}^{R} \mid u_{1}, u_{2},\right. \\
\left.u_{3}, u_{4} \in \Sigma_{D N A}^{*}\right\}
\end{array}
$$

can be generated by a matrix insertion-deletion system.

Proof. The language $L_{h s}$ can be generated by matrix insertion-deletion system

$$
\begin{aligned}
\Upsilon_{h s}=\left(\left\{b, \bar{b}, \dagger_{1}, \dagger_{2}, \dagger_{3}, \dagger_{4}, \dagger_{5}, \bullet\right\},\{b, \bar{b}, \bullet\},\right. \\
\left.\left\{\dagger_{1} \bullet \dagger_{2} \bullet \dagger_{3} \bullet \dagger_{4} \bullet \dagger_{5}, \bullet \bullet \bullet\right\}, R\right)
\end{aligned}
$$

where $b \in\{a, t, g, c\}, \bar{b}$ is a complement of $b$ and $R$ is 
given as

$$
\begin{aligned}
R_{I_{1}} & =\left[\left(\dagger_{1}, \lambda / b, \lambda\right),\left(\lambda, \lambda / \bar{b}, \dagger_{2}\right)\right], \\
R_{I_{2}} & =\left[\left(\dagger_{2}, \lambda / b, \lambda\right),\left(\lambda, \lambda / \bar{b}, \dagger_{3}\right)\right], \\
R_{I_{3}} & =\left[\left(\dagger_{3}, \lambda / b, \lambda\right),\left(\lambda, \lambda / \bar{b}, \dagger_{4}\right)\right], \\
R_{I_{4}} & =\left[\left(\dagger_{4}, \lambda / b, \lambda\right),\left(\lambda, \lambda / \bar{b}, \dagger_{5}\right)\right], \\
R_{D_{1}} & =\left[\left(\lambda, \dagger_{1} / \lambda, \lambda\right)\right], \\
R_{D_{2}} & =\left[\left(\lambda, \dagger_{2} / \lambda, \lambda\right)\right], \\
R_{D_{3}} & =\left[\left(\lambda, \dagger_{3} / \lambda, \lambda\right)\right], \\
R_{D_{4}} & =\left[\left(\lambda, \dagger_{4} / \lambda, \lambda\right)\right], \\
R_{D_{5}} & =\left[\left(\lambda, \dagger_{5} / \lambda, \lambda\right)\right] .
\end{aligned}
$$

A sample derivation is given as follows:

$$
\begin{aligned}
& \dagger_{1}^{\downarrow} \bullet \downarrow \dagger_{2} \bullet \dagger_{3} \bullet \dagger_{4} \bullet \dagger_{5} \\
& \Longrightarrow R_{I_{1}} \dagger_{1}^{\downarrow} \underline{a} \bullet \underline{t}^{\dagger} \dagger_{2} \bullet \dagger_{3} \bullet \dagger_{4} \bullet \dagger_{5} \\
& \Longrightarrow R_{I_{1}} \dagger_{1} \underline{c} a \bullet t \underline{g} \dagger_{2}^{\downarrow} \bullet \downarrow \dagger_{3} \bullet \dagger_{4} \bullet \dagger_{5} \\
& \Longrightarrow R_{I_{2}} \dagger_{1} c a \bullet \operatorname{tg} \dagger_{2}^{\downarrow} \underline{a} \bullet{ }^{\downarrow} \underline{t} \dagger_{3} \bullet \dagger_{4} \bullet \dagger_{5} \\
& \Longrightarrow R_{I_{2}} \dagger_{1} c a \bullet t g \dagger_{2} \underline{c} a \bullet t \underline{g} \dagger_{3}^{\downarrow} \bullet \downarrow \dagger_{4} \bullet \dagger_{5} \\
& \Longrightarrow R_{I_{3}} \dagger_{1} c a \bullet t g \dagger_{2} c a \bullet t g \dagger_{3}^{\downarrow} \underline{g} \bullet{ }^{\downarrow} \underline{c} \dagger_{4} \bullet \dagger_{5} \\
& \Longrightarrow R_{I_{3}} \dagger_{1} c a \bullet t g \dagger_{2} c a \bullet t g \dagger_{3} \underline{a} g \bullet c \underline{t} \dagger_{4}^{\downarrow} \bullet \downarrow \dagger_{5} \\
& \Longrightarrow R_{I_{4}} \dagger_{1} c a \bullet t g \dagger_{2} c a \bullet t g \dagger_{3} a g \bullet c t \dagger_{4}^{\downarrow} \underline{a} \bullet \underline{t}^{\downarrow} \dagger_{5} \\
& \Longrightarrow R_{I_{4}} \dagger_{1} c a \bullet \mathrm{tg}_{\dagger_{2}} \mathrm{ca} \bullet \mathrm{tg} \dagger_{3} \mathrm{ag} \bullet \mathrm{ct} \dagger_{4} \underline{\mathrm{c} a} \bullet \mathrm{tg} \dagger_{5} \\
& \Longrightarrow R_{D_{1}}{ }^{\Downarrow} \mathrm{ca} \bullet \mathrm{tg} \dagger_{2} \mathrm{ca} \bullet \mathrm{tg} \dagger_{3} \mathrm{ag} \bullet \mathrm{ct} \dagger_{4} \mathrm{ca} \bullet \mathrm{tg} \dagger_{5} \\
& \Longrightarrow R_{D_{2}} \mathrm{ca} \bullet \mathrm{tg}^{\Downarrow} \mathrm{ca} \bullet \mathrm{tg} \dagger_{3} \mathrm{ag} \bullet \mathrm{ct} \dagger_{4} \mathrm{ca} \bullet \mathrm{tg} \dagger_{5} \\
& \Longrightarrow R_{D_{3}} \mathrm{ca} \bullet \mathrm{tgca} \bullet \mathrm{tg}^{\Downarrow} \mathrm{ag} \bullet \mathrm{ct} \dagger_{4} \mathrm{ca} \bullet \mathrm{tg} \dagger_{5} \\
& \Longrightarrow R_{D_{4}} c a \bullet t g c a \bullet t g a g \bullet c t^{\Downarrow} c a \bullet t g \dagger_{5} \\
& \Longrightarrow R_{D_{5}} \mathrm{ca} \bullet \mathrm{tgca} \bullet \mathrm{tgag} \bullet \mathrm{ctca} \bullet \mathrm{tg}^{\Downarrow} \text {. }
\end{aligned}
$$

\begin{tabular}{|c|c|}
\hline Fig. no. & $\begin{array}{c}\text { Bio-molecular structure } \\
\text { Formal language representation }\end{array}$ \\
\hline 4(a) & $\begin{array}{c}\text { Internal loop } \\
L_{i l}=\left\{u_{1} v_{1} u_{2} v_{3}{\overline{u_{2}}}^{R} v_{2}{\overline{u_{1}}}^{R}\right\}\end{array}$ \\
\hline 4(b) & $\begin{array}{c}\text { Bulge loop } \\
L_{b l}=\left\{u_{1} v_{1} u_{2} v_{2}{\overline{u_{2}}}^{R}{\overline{u_{1}}}^{R}\right\}\end{array}$ \\
\hline 5(a) & $\begin{array}{c}\text { Multi branch loop } \\
L_{m b l}=\left\{u_{1} v_{1}{\overline{v_{1}}}^{R} u_{2} \overline{u_{2}}{ }^{R} v_{2}{\overline{u_{1}}}^{R}\right\}\end{array}$ \\
\hline $5(\mathrm{~b})$ & $\begin{array}{c}\text { Extended pseudoknot } \\
L_{e p k}=\left\{u_{1} v_{1}{\overline{u_{1}}}^{R} u_{2}{\overline{v_{1}}}^{R}{\overline{u_{2}}}^{R}\right\}\end{array}$ \\
\hline 6 & $\begin{array}{c}\text { Kissing hairpin } \\
L_{k h p}=\left\{u_{1} v_{1} \#_{1} v_{2} u_{2} \overline{u_{2}}{ }^{R} v_{3} \#_{2} v_{4} \overline{u_{1}}{ }^{R}\right\} \\
\end{array}$ \\
\hline 7 (a) & $\begin{array}{c}\text { Simple H-type } \\
L_{s h t}=\left\{u_{1} v_{1} u_{2} \overline{u_{1}}{ }^{R} v_{2}{\overline{u_{2}}}^{R}\right\}\end{array}$ \\
\hline 7(b) & $\begin{array}{c}\text { Recursive pseudoknot } \\
L_{r p s}=\left\{u_{1} u_{2} u_{3}{\overline{u_{2}}}^{R} u_{4}{\overline{u_{1}}}^{R}{\overline{u_{4}}}^{R} u_{5}{\overline{u_{5}}}^{R}{\overline{u_{3}}}^{R}\right\}\end{array}$ \\
\hline 8 & $\begin{array}{c}\text { Three-knot structure } \\
L_{t k s}=\left\{u_{1} v u_{2} u_{3} \overline{u_{1}}{ }^{R} \overline{u_{2}}{ }^{R} \overline{u_{3}}{ }^{R}\right\}\end{array}$ \\
\hline
\end{tabular}

\section{RNA secondary structures}

In this section, first we show the interpretation of various RNA secondary structures in terms of formal language representations (as shown in Table 2), and we model such structures using matrix insertion-deletion systems. If the strings are collected as per the dotted directed lines, the RNA secondary structures represented in Figs. 4(a), 4(b), $5(\mathrm{a}), 5(\mathrm{~b}), 6,7(\mathrm{a}), 7(\mathrm{~b})$ and 8 can be given in terms of languages as shown in Table 2 .

Thus, given a DNA/RNA sequence, we can (try to) first identify the corresponding the formal language and then one can think of what matrix insertion-deletion system can generate the language.

Lemma 9. The internal loop structure (see Fig. 4(a))

$$
L_{i l}=\left\{u_{1} v_{1} u_{2} v_{3}{\overline{u_{2}}}^{R} v_{2}{\overline{u_{1}}}^{R} \mid u_{1}, u_{2}, v_{1}, v_{2}, v_{3} \in \Sigma_{R N A}^{*}\right\}
$$

can be generated by a matrix insertion-deletion system.
Table 2. Formal language representation: $\#_{1}$ represents the $A \bar{A}$ and $\#_{2}$ represents the $B \bar{B}$ in Fig. 8.

Proof. The language $L_{i l}$ can be generated by the matrix insertion-deletion system

$$
\Upsilon_{i l}=\left(\left\{b, \bar{b}, \dagger_{1}, \dagger_{2}, \dagger_{3}, \dagger_{4}\right\},\{b, \bar{b}\},\left\{\dagger_{1} \dagger_{3} \dagger_{4} \dagger_{2}\right\}, R\right),
$$

where $b \in\{a, u, g, c\}, \bar{b}$ is the complement of $b$ and $R$ is given as follows:

$$
\begin{aligned}
R_{I_{1}} & =\left[\left(\lambda, \lambda / b, \dagger_{1}\right),\left(\dagger_{2}, \lambda / \bar{b}, \lambda\right)\right], \\
R_{I_{2}} & =\left[\left(\lambda, \lambda / b, \dagger_{3}\right),\left(\dagger_{4}, \lambda / \bar{b}, \lambda\right)\right], \\
R_{I_{3}} & =\left[\left(\dagger_{1}, \lambda / b, \lambda\right)\right], \\
R_{I_{4}} & =\left[\left(\dagger_{3}, \lambda / b, \lambda\right)\right], \\
R_{I_{5}} & =\left[\left(\lambda, \lambda / b, \dagger_{2}\right)\right], \\
R_{D_{1}} & =\left[\left(\lambda, \dagger_{1} / \lambda, \lambda\right)\right], \\
R_{D_{2}} & =\left[\left(\lambda, \dagger_{2} / \lambda, \lambda\right)\right], \\
R_{D_{3}} & =\left[\left(\lambda, \dagger_{3} / \lambda, \lambda\right)\right], \\
R_{D_{4}} & =\left[\left(\lambda, \dagger_{4} / \lambda, \lambda\right)\right] .
\end{aligned}
$$

A sample derivation is given as follows:

$$
\begin{aligned}
& \downarrow \dagger_{1} \dagger_{3} \dagger_{4} \dagger_{2}^{\downarrow} \Longrightarrow_{R_{I_{1}}} \underline{a}^{\downarrow} \dagger_{1} \dagger_{3} \dagger_{4} \dagger_{2}^{\downarrow} \underline{u} \\
& \Longrightarrow_{R_{I_{1}}} a \underline{u} \dagger_{1}^{\downarrow} \dagger_{3} \dagger_{4}^{\downarrow} \dagger_{2} \underline{a} u \\
& \Longrightarrow_{R_{I_{2}}} a u \dagger_{1} \underline{u}^{\downarrow} \dagger_{3} \dagger_{4}^{\downarrow} \underline{a} \dagger_{2} a u \\
& \Longrightarrow_{R_{I_{2}}} a u \dagger_{1}^{\downarrow} u \underline{a} \dagger_{3} \dagger_{4} \underline{u} a \dagger_{2} a u \\
& \Longrightarrow_{R_{I_{3}}} a u \dagger_{1}^{\downarrow} \underline{g} u a \dagger_{3} \dagger_{4} u a \dagger_{2} a u \\
& \Longrightarrow_{R_{I_{3}}} a u \dagger_{1} \underline{c} g u a \dagger_{3}^{\downarrow} \dagger_{4} u a \dagger_{2} a u
\end{aligned}
$$


$\Longrightarrow R_{I_{4}} a u \dagger_{1} c g u a \dagger_{3}^{\downarrow} \underline{c} \dagger_{4} u a \dagger_{2} a u$

$\Longrightarrow R_{I_{4}} a u \dagger_{1} c g u a \dagger_{3} g_{c} \dagger_{4} u a \dagger_{2} a u$

$\Longrightarrow R_{D_{1}} a u^{\Downarrow} \mathrm{cgua}_{3} \mathrm{gc}_{4} u a \dagger_{2} \mathrm{au}$

$\Longrightarrow_{R_{D_{2}}}$ aucgua $\dagger_{3} g_{c} \dagger_{4} u a^{\Downarrow} a u$

$\Longrightarrow R_{D_{3}}$ aucgua $\mathrm{gc}^{\Downarrow} \dagger_{4} u a a u$

$\Longrightarrow R_{D_{4}}$ aucguagc ${ }^{\Downarrow} u a a u$.

The idea is that $\dagger_{1}, \dagger_{2}, \dagger_{3}$ and $\dagger_{4}$ are used as markers. $\dagger_{1}$ and $\dagger_{2}$ are used to control the $u_{1} \bar{u}_{1}^{R}$ part of the language. Whenever a $b$ is adjoined to the left of $\dagger_{1}$, its corresponding complementary $\bar{b}$ is adjoined to the right of $\dagger_{2}$ and the synchronization is maintained. Similarly, $\dagger_{3}$ and $\dagger_{4}$ are used to control the $u_{2} \overline{u_{2}}{ }^{R}$ part of the language, $\dagger_{1}, \dagger_{2}$ and $\dagger_{3}$ are used to control the $v_{1}, v_{2}$ and $v_{3}$ part of the language, respectively.

Lemma 10. The bulge loop structure (see Fig. 4(b))

$$
L_{b l}=\left\{u_{1} v_{1} u_{2} v_{2}{\overline{u_{2}}}^{R}{\overline{u_{1}}}^{R} \mid u_{1}, u_{2}, v_{1}, v_{2} \in \Sigma_{R N A}^{*}\right\}
$$

can be generated by a matrix insertion-deletion system.

Proof. The language $L_{b l}$ can be generated by the matrix insertion-deletion system

$$
\Upsilon_{b l}=\left(\left\{b, \bar{b}, \dagger_{1}, \dagger_{2}, \dagger_{3}, \dagger_{4}\right\},\{b, \bar{b}\},\left\{\dagger_{1} \dagger_{4} \dagger_{3} \dagger_{2}\right\}, R\right),
$$

where $b \in\{a, u, g, c\}, \bar{b}$ is the complement of $b$ and $R$ is given as follows:

$$
\begin{aligned}
R_{I_{1}} & =\left[\left(\lambda, \lambda / b, \dagger_{1}\right),\left(\dagger_{2}, \lambda / \bar{b}, \lambda\right)\right], \\
R_{I_{2}} & =\left[\left(\lambda, \lambda / b, \dagger_{4}\right),\left(\dagger_{3}, \lambda / \bar{b}, \lambda\right)\right], \\
R_{I_{3}} & =\left[\left(\dagger_{1}, \lambda / b, \lambda\right)\right], \\
R_{I_{4}} & =\left[\left(\dagger_{4}, \lambda / b, \lambda\right)\right], \\
R_{D_{1}} & =\left[\left(\lambda, \dagger_{1} / \lambda, \lambda\right),\right. \\
R_{D_{2}} & =\left[\left(\lambda, \dagger_{2} / \lambda, \lambda\right)\right], \\
R_{D_{3}} & =\left[\left(\lambda, \dagger_{3} / \lambda, \lambda\right)\right], \\
R_{D_{4}} & =\left[\left(\lambda, \dagger_{4} / \lambda, \lambda\right)\right] .
\end{aligned}
$$

As the derivation and the language are similar to internal loop structure, we omit the sample derivation.

Lemma 11. The multi-branch loop structure (see Fig. 5(a))

$$
L_{m b l}=\left\{u_{1} v_{1}{\overline{v_{1}}}^{R} u_{2}{\overline{u_{2}}}^{R} \bar{v}_{2}{\overline{u_{1}}}^{R} \mid u_{1}, u_{2}, v_{1}, v_{2} \in \Sigma_{R N A}^{*}\right\}
$$

can be generated by a matrix insertion-deletion system.

Proof. The language $L_{m b l}$ can be generated by the matrix insertion-deletion system

$$
\Upsilon_{m b l}=\left(\left\{b, \bar{b}, \dagger_{1}, \dagger_{2}, \dagger_{3}, \dagger_{4}\right\},\{b, \bar{b}\},\left\{\dagger_{1} \dagger_{3} \dagger_{4} \dagger_{2}\right\}, R\right),
$$

where $b \in\{a, u, g, c\}, \bar{b}$ is complement of $b$ and $R$ is given as follows:

$$
\begin{aligned}
R_{I_{1}} & =\left[\left(\lambda, \lambda / b, \dagger_{1}\right),\left(\dagger_{2}, \lambda / \bar{b}, \lambda\right)\right], \\
R_{I_{2}} & =\left[\left(\lambda, \lambda / b, \dagger_{4}\right),\left(\dagger_{4}, \lambda / \bar{b}, \lambda\right)\right], \\
R_{I_{3}} & =\left[\left(\lambda, \lambda / b, \dagger_{3}\right),\left(\dagger_{3}, \lambda / \bar{b}, \lambda\right)\right], \\
R_{I_{4}} & =\left[\left(\lambda, \lambda / b, \dagger_{2}\right)\right], \\
R_{D_{1}} & =\left[\left(\lambda, \dagger_{1} / \lambda, \lambda\right)\right], \\
R_{D_{2}} & =\left[\left(\lambda, \dagger_{2} / \lambda, \lambda\right)\right], \\
R_{D_{3}} & =\left[\left(\lambda, \dagger_{3} / \lambda, \lambda\right)\right], \\
R_{D_{4}} & =\left[\left(\lambda, \dagger_{4} / \lambda, \lambda\right)\right] .
\end{aligned}
$$

As the derivation and the language are similar to internal loop structure, we omit the sample derivation.

Lemma 12. The extended pseudoknot structure (see Fig. 5(b))

$$
L_{e p k}=\left\{u_{1} v_{1} \overline{u_{1}}{ }^{R} u_{2} \overline{v_{1}} \bar{u}_{\overline{u_{2}}}^{R} \mid u_{1}, u_{2}, v_{1} \in \Sigma_{R N A}^{*}\right\}
$$

can be generated by a matrix insertion-deletion system.

Proof. The language $L_{e p k}$ can be generated by the matrix insertion-deletion system

$$
\Upsilon_{e p k}=\left(\left\{b, \bar{b}, \dagger_{1}, \dagger_{2}, \dagger_{3}, \dagger_{4}\right\},\{b, \bar{b}\},\left\{\dagger_{1} \dagger_{2} \dagger_{3} \dagger_{4}\right\}, R\right),
$$

where $b \in\{a, u, g, c\}, \bar{b}$ is a complement of $b$ and $R$ is given as follows:

$$
\begin{aligned}
R_{I_{1}} & =\left[\left(\lambda, \lambda / b, \dagger_{1}\right),\left(\dagger_{2}, \lambda / \bar{b}, \lambda\right)\right], \\
R_{I_{2}} & =\left[\left(\dagger_{1}, \lambda / b, \lambda\right),\left(\dagger_{3}, \lambda / \bar{b}, \lambda\right)\right], \\
R_{I_{3}} & =\left[\left(\lambda, \lambda / b, \dagger_{3}\right),\left(\dagger_{4}, \lambda / \bar{b}, \lambda\right)\right], \\
R_{D_{1}} & =\left[\left(\lambda, \dagger_{1} / \lambda, \lambda\right)\right], \\
R_{D_{2}} & =\left[\left(\lambda, \dagger_{2} / \lambda, \lambda\right)\right], \\
R_{D_{3}} & =\left[\left(\lambda, \dagger_{3} / \lambda, \lambda\right)\right], \\
R_{D_{4}} & =\left[\left(\lambda, \dagger_{4} / \lambda, \lambda\right)\right] .
\end{aligned}
$$

A sample derivation is given as follows:

$$
\begin{aligned}
& \downarrow \dagger_{1} \dagger_{2}^{\downarrow} \dagger_{3} \dagger_{4} \Longrightarrow_{R_{I_{1}}} \underline{a}^{\downarrow} \dagger_{1} \dagger_{2} \underline{u} \dagger_{3} \dagger_{4} \\
& \Longrightarrow_{R_{I_{1}}} a \underline{g}^{\downarrow} \dagger_{1} \dagger_{2}^{\downarrow} \underline{c} u \dagger_{3} \dagger_{4} \\
& \Longrightarrow R_{I_{1}} a g \underline{c} \dagger_{1}^{\downarrow} \dagger_{2} \underline{g c u} \dagger_{3}^{\downarrow} \dagger_{4} \\
& \Longrightarrow R_{I_{2}} a g c \dagger_{1}^{\downarrow} \underline{a} \dagger_{2} g c u \dagger_{3}^{\downarrow} \underline{u} \dagger_{4} \\
& \Longrightarrow_{R_{I_{2}}} a g c \dagger_{1} \underline{g} a \dagger_{2} g c u^{\downarrow} \dagger_{3} \underline{c u} \dagger_{4}^{\downarrow} \\
& \Longrightarrow_{R_{I_{3}}} a g c \dagger_{1} g a \dagger_{2} g c u \underline{a}^{\downarrow} \dagger_{3} c u \dagger_{4}^{\downarrow} \underline{u} \\
& \Longrightarrow_{R_{I_{3}}} a g c \dagger_{1} g a \dagger_{2} g c u a \underline{c} \dagger_{3} c u \dagger_{4} \underline{g u} \\
& \Longrightarrow_{R_{D_{1}}} a g c^{\Downarrow} g a \dagger_{2} g c u a c \dagger_{3} c u \dagger_{4} g u \\
& \Longrightarrow_{R_{D_{2}}} \text { agcga }^{\Downarrow} \text { gcuac }_{3} \mathrm{cu} \dagger_{4} \mathrm{gu} \\
& \Longrightarrow R_{D_{3}} \text { agcgagcuac }^{\Downarrow} \mathrm{cu} \dagger_{4} \mathrm{gu} \\
& \Longrightarrow R_{D_{4}} \text { agcgagcuaccu }{ }^{\Downarrow} \text { gu }
\end{aligned}
$$


Lemma 13. The kissing hairpin structure (see Fig. 6)

$$
\begin{array}{r}
L_{k h p}=\left\{u_{1} v_{1} A \bar{A} v_{2} u_{2}{\overline{u_{2}}}^{R} v_{3} B \bar{B} v_{4}{\overline{u_{1}}}^{R} \mid u_{1}, u_{2}, v_{1},\right. \\
\left.v_{2}, v_{3}, v_{4} \in \Sigma_{R N A}^{*} \text { and } A, B \in \Sigma_{R N A}\right\}
\end{array}
$$

can be generated by a matrix insertion-deletion system.

Proof. The language $L_{k h p}$ can be generated by the matrix insertion-deletion system

$$
\begin{aligned}
\Upsilon_{k h p}=\left(\left\{b, \bar{b}, \dagger_{1}, \dagger_{2}, \dagger_{3}, \dagger_{4}\right\},\{b, \bar{b}\}\right. & ,\left\{\dagger_{1} A \bar{A}\right. \\
& \left.\left.\dagger_{3} \dagger_{4} B \bar{B} \dagger_{2}\right\}, R\right),
\end{aligned}
$$

where $A, B, b \in\{a, u, g, c\}, \bar{A}, \bar{B}, \bar{b}$ is the complement of $A, B, b$ and $R$ is given as follows:

$$
\begin{aligned}
R_{I_{1}} & =\left[\left(\lambda, \lambda / b, \dagger_{1}\right),\left(\dagger_{2}, \lambda / \bar{b}, \lambda\right)\right], \\
R_{I_{2}} & =\left[\left(\dagger_{3}, \lambda / b, \lambda\right),\left(\lambda, \lambda / \bar{b}, \dagger_{4}\right)\right], \\
R_{I_{3}} & =\left[\left(\dagger_{1}, \lambda / b, \lambda\right)\right], \\
R_{I_{4}} & =\left[\left(\lambda, \lambda / b, \dagger_{3}\right)\right], \\
R_{I_{5}} & =\left[\left(\lambda, \lambda / b, \dagger_{2}\right)\right], \\
R_{I_{6}} & =\left[\left(\dagger_{4}, \lambda / b, \lambda\right)\right], \\
R_{D_{1}} & =\left[\left(\lambda, \dagger_{1} / \lambda, \lambda\right)\right], \\
R_{D_{2}} & =\left[\left(\lambda, \dagger_{2} / \lambda, \lambda\right)\right], \\
R_{D_{3}} & =\left[\left(\lambda, \dagger_{3} / \lambda, \lambda\right)\right], \\
R_{D_{4}} & =\left[\left(\lambda, \dagger_{4} / \lambda, \lambda\right)\right] .
\end{aligned}
$$

A sample derivation is given as follows:

$$
\begin{aligned}
& { }^{\downarrow} \dagger_{1} a u \dagger_{3} \dagger_{4} u a \dagger_{2}^{\downarrow} \Longrightarrow R_{I_{1}} \underline{c}^{\downarrow} \dagger_{1} a u \dagger_{3} \dagger_{4} u a \dagger_{2}^{\downarrow} \underline{g} \\
& \Longrightarrow R_{I_{1}} \underline{c u}_{\dagger_{1}} \mathrm{au} \dagger_{3} \downarrow \downarrow \dagger_{4} u a \dagger_{2} \underline{a} g \\
& \Longrightarrow_{R_{I_{2}}} \mathrm{cu} \dagger_{1}^{\downarrow} \mathrm{au} \dagger_{3} \underline{\mathrm{c}} \underline{\mathrm{g}} \dagger_{4} u a \dagger_{2} a g \\
& \Longrightarrow R_{I_{3}} c u \dagger_{1} g_{a}{ }^{\downarrow} \dagger_{3} c g \dagger_{4} u a \dagger_{2} a g \\
& \Longrightarrow_{R_{I_{4}}} c u \dagger_{1} g a u \underline{u} \dagger_{3} c g \dagger_{4} u a^{\downarrow} \dagger_{2} a g \\
& \Longrightarrow R_{I_{5}} c u \dagger_{1} \text { gauu } \dagger_{3} c g \dagger_{4}^{\downarrow} u a \underline{c} \dagger_{2} a g \\
& \Longrightarrow R_{I_{6}} c u \dagger_{1} \text { gauu } \dagger_{3} c g \dagger_{4} \underline{g u a c}_{2} \mathrm{ag} \\
& \Longrightarrow R_{D_{1}} \mathrm{cu}^{\Downarrow} \mathrm{gauu} \dagger_{3} \mathrm{cg} \dagger_{4} \mathrm{guac}_{2} \mathrm{ag} \\
& \Longrightarrow R_{D_{2}} \text { cugauu } \dagger_{3} \mathrm{cg} \dagger_{4} \mathrm{guac}^{\Downarrow} \mathrm{ag} \\
& \Longrightarrow R_{D_{3}} \text { cugauu }^{\Downarrow} \mathrm{cg}_{4} \dagger_{4} \text { guacag } \\
& \Longrightarrow R_{D_{4}} \text { cugauucg }{ }^{\Downarrow} \text { guacag. }
\end{aligned}
$$

The idea for generating the language $L_{k h p}$ is given as follows. As $A \bar{A}$ and $B \bar{B}$ are already present in the axiom, markers are not required to generate it. To generate the remaining part of the language, $\dagger_{1}, \dagger_{2}, \dagger_{3}$ and $\dagger_{4}$ are used as markers. $\dagger_{1}$ and $\dagger_{2}$ are used to control the $u_{1}{\overline{u_{1}}}^{R}$ part of the language. Whenever a $b$ is adjoined to the left of $\dagger_{1}$, its corresponding complementary $\bar{b}$ is adjoined to the right of $\dagger_{2}$ and the synchronization is maintained. Similarly, $\dagger_{3}$ and $\dagger_{4}$ are used to control the $u_{2}{\overline{u_{2}}}^{R}$ part of the language, $\dagger_{1}, \dagger_{3}, \dagger_{4}$ and $\dagger_{2}$ are used to control the $v_{1}, v_{2}, v_{3}$ and $v_{4}$ part of the language, respectively.
Lemma 14. The simple H-type structure (see Fig. 7(a))

$$
L_{s h t}=\left\{u_{1} v_{1} u_{2}{\overline{u_{1}}}^{R} v_{2} \overline{u_{2}}{ }^{R} \mid u_{1}, u_{2}, v_{1}, v_{2} \in \Sigma_{R N A}^{*}\right\}
$$

can be generated by a matrix insertion-deletion system.

Proof. The language $L_{\text {sht }}$ can be generated by the matrix insertion-deletion system

$$
\Upsilon_{s h t}=\left(\left\{b, \bar{b}, \dagger_{1}, \dagger_{2}, \dagger_{3}\right\},\{b, \bar{b}\},\left\{\dagger_{1} \dagger_{2} \dagger_{3}\right\}, R\right),
$$

where $b \in\{a, u, g, c\}, \bar{b}$ is a complement of $b$ and $R$ is given as follows:

$$
\begin{aligned}
R_{I_{1}} & =\left[\left(\lambda, \lambda / b, \dagger_{1}\right),\left(\dagger_{2}, \lambda / \bar{b}, \lambda\right)\right], \\
R_{I_{2}} & =\left[\left(\lambda, \lambda / b, \dagger_{2}\right),\left(\dagger_{3}, \lambda / \bar{b}, \lambda\right)\right], \\
R_{I_{3}} & =\left[\left(\dagger_{1}, \lambda / b, \lambda\right)\right], \\
R_{I_{4}} & =\left[\left(\lambda, \lambda / b, \dagger_{3}\right)\right], \\
R_{D_{1}} & =\left[\left(\lambda, \dagger_{1} / \lambda, \lambda\right)\right], \\
R_{D_{2}} & =\left[\left(\lambda, \dagger_{2} / \lambda, \lambda\right)\right], \\
R_{D_{3}} & =\left[\left(\lambda, \dagger_{3} / \lambda, \lambda\right)\right] .
\end{aligned}
$$

As the derivation and the language are similar to extended pseudoknot structure, we omit the sample derivation.

Lemma 15. The recursive pseudoknot structure (see Fig. 7(b))

$$
\begin{array}{r}
L_{r p s}=\left\{u_{1} u_{2} u_{3}{\overline{u_{2}}}^{R} u_{4}{\overline{u_{1}}}^{R}{\overline{u_{4}}}^{R} u_{5}{\overline{u_{5}}}^{R}{\overline{u_{3}}}^{R} \mid u_{1}, u_{2},\right. \\
\left.u_{3}, u_{4}, u_{5} \in \Sigma_{R N A}^{*}\right\}
\end{array}
$$

can be modelled using a matrix insertion-deletion system.

Proof. The language $L_{r p s}$ can be generated by the matrix insertion-deletion system

$\Upsilon_{r p s}=\left(\left\{b, \bar{b}, \dagger_{1}, \dagger_{2}, \dagger_{3}, \dagger_{4} \dagger_{5}\right\},\{b, \bar{b}\},\left\{\dagger_{1} \dagger_{2} \dagger_{3} \dagger_{4} \dagger_{5}\right\}, R\right)$,

where $b \in\{a, u, g, c\}, \bar{b}$ is a complement of $b$ and $R$ is given as follows:

$$
\begin{aligned}
R_{I_{1}} & =\left[\left(\lambda, \lambda / b, \dagger_{1}\right),\left(\dagger_{3}, \lambda / \bar{b}, \lambda\right)\right], \\
R_{I_{2}} & =\left[\left(\dagger_{1}, \lambda / b, \lambda\right),\left(\dagger_{2}, \lambda / \bar{b}, \lambda\right)\right], \\
R_{I_{3}} & =\left[\left(\lambda, \lambda / b, \dagger_{2}\right),\left(\dagger_{5}, \lambda / \bar{b}, \lambda\right)\right], \\
R_{I_{4}} & =\left[\left(\lambda, \lambda / b, \dagger_{3}\right),\left(\lambda, \lambda / \bar{b}, \dagger_{4}\right)\right], \\
R_{I_{5}} & =\left[\left(\dagger_{4}, \lambda / b, \lambda\right),\left(\lambda, \lambda / \bar{b}, \dagger_{5}\right)\right], \\
R_{D_{1}} & =\left[\left(\lambda, \dagger_{1} / \lambda, \lambda\right)\right], \\
R_{D_{2}} & =\left[\left(\lambda, \dagger_{2} / \lambda, \lambda\right)\right], \\
R_{D_{3}} & =\left[\left(\lambda, \dagger_{3} / \lambda, \lambda\right)\right], \\
R_{D_{4}} & =\left[\left(\lambda, \dagger_{4} / \lambda, \lambda\right)\right], \\
R_{D_{5}} & =\left[\left(\lambda, \dagger_{5} / \lambda, \lambda\right)\right] .
\end{aligned}
$$


A sample derivation is given as follows:

$$
\begin{aligned}
& \downarrow_{1} \dagger_{2} \dagger_{3}^{\downarrow} \dagger_{4} \dagger_{5} \Longrightarrow R_{I_{1}} \underline{a}^{\downarrow} \dagger_{1} \dagger_{2} \dagger_{3}^{\downarrow} \underline{u} \dagger_{4} \dagger_{5} \\
& \Longrightarrow R_{I_{1}} \underline{a} u \dagger_{1}^{\downarrow} \dagger_{2}^{\downarrow} \dagger_{3} \underline{a} u \dagger_{4} \dagger_{5} \\
& \Longrightarrow_{R_{I_{2}}} \text { au } \dagger_{1}^{\downarrow} \underline{g} \dagger_{2}^{\downarrow} \underline{c} \dagger_{3} a u \dagger_{4} \dagger_{5} \\
& \Longrightarrow R_{I_{2}} a u \dagger_{1} \underline{u} g^{\downarrow} \dagger_{2} \underline{a} c \dagger_{3} a u \dagger_{4} \dagger_{5}^{\downarrow} \\
& \Longrightarrow_{R_{I_{3}}} a u \dagger_{1} u g \underline{u}^{\downarrow} \dagger_{2} a c \dagger_{3} a u \dagger_{4} \dagger_{5}^{\downarrow} \underline{a} \\
& \Longrightarrow R_{I_{3}} a u \dagger_{1} u g u \underline{c} \dagger_{2} a c^{\downarrow} \dagger_{3} a u^{\downarrow} \dagger_{4} \dagger_{5} \underline{g} a \\
& \Longrightarrow R_{I_{4}} a u \dagger_{1} u g u c \dagger_{2} a c \underline{a}^{\downarrow} \dagger_{3} a u \underline{u}^{\downarrow} \dagger_{4} \dagger_{5} g a \\
& \Longrightarrow R_{I_{4}} \text { au } \dagger_{1} \text { uguc } \dagger_{2} \text { aca } \underline{u} \dagger_{3} \text { auu } \underline{a}_{4}{ }_{4}{ }^{\downarrow} \dagger_{5} g a \\
& \Longrightarrow_{R_{I_{5}}} \text { au } \dagger_{1} \text { uguc } \dagger_{2} \text { acau } \dagger_{3} \text { auua } \dagger_{4}{ }^{\downarrow} \underline{g}^{\downarrow}{ }^{\downarrow} \dagger_{5} g a \\
& \Longrightarrow R_{I_{5}} \text { au } \dagger_{1} \text { uguc } \dagger_{2} \text { acau } \dagger_{3} \text { auua } \dagger_{4} \underline{u} g c \underline{a} \dagger_{5} g a \\
& \Longrightarrow R_{D_{1}} a u^{\Downarrow} u g u c \dagger_{2} \text { acau } \dagger_{3} \text { auua } \dagger_{4} \text { ugca } \dagger_{5} g a \\
& \Longrightarrow_{R_{D_{2}}} \text { auuguc }{ }^{\Downarrow} \text { acau } \dagger_{3} \text { auua } \dagger_{4} \text { ugca } \dagger_{5} g a \\
& \Longrightarrow R_{D_{3}} \text { auugucacau }{ }^{\Downarrow} \text { auua } \dagger_{4} \text { ugca } \dagger_{5} g a \\
& \Longrightarrow R_{D_{4}} \text { auugucacauauua }{ }^{\Downarrow} \text { uca } \dagger_{5} g a \\
& \Longrightarrow R_{D_{5}} \text { auugucacauauuaugca }{ }^{\Downarrow} \text { ga. }
\end{aligned}
$$

Lemma 16. The three-knot structure (see Fig. 8)

$$
L_{t k s}=\left\{u_{1} v u_{2} u_{3}{\overline{u_{1}}}^{R}{\overline{u_{2}}}^{R}{\overline{u_{3}}}^{R} \mid u_{1}, u_{2}, u_{3}, v \in \Sigma_{R N A}^{*}\right\}
$$

can be generated by a matrix insertion-deletion system.

Proof. The language $L_{t k s}$ can be generated by the matrix insertion-deletion system

$$
\Upsilon_{t k s}=\left(\left\{b, \bar{b}, \dagger_{1}, \dagger_{2}, \dagger_{3}, \dagger_{4}\right\},\{b, \bar{b}\},\left\{\dagger_{1} \dagger_{2} \dagger_{3} \dagger_{4}\right\}, R\right),
$$

where $b \in\{a, u, g, c\}, \bar{b}$ is the complement of $b$ and $R$ is given as follows:

$$
\begin{aligned}
R_{I_{1}} & =\left[\left(\lambda, \lambda / b, \dagger_{1}\right),\left(\lambda, \lambda / \bar{b}, \dagger_{3}\right)\right], \\
R_{I_{2}} & =\left[\left(\lambda, \lambda / b, \dagger_{2}\right),\left(\dagger_{3}, \lambda / \bar{b}, \lambda\right)\right], \\
R_{I_{3}} & =\left[\left(\dagger_{2}, \lambda / b, \lambda\right),\left(\lambda, \lambda / \bar{b}, \dagger_{4}\right)\right], \\
R_{I_{4}} & =\left[\left(\dagger_{1}, \lambda / b, \lambda\right)\right], \\
R_{D_{1}} & =\left[\left(\lambda, \dagger_{1} / \lambda, \lambda\right)\right], \\
R_{D_{2}} & =\left[\left(\lambda, \dagger_{2} / \lambda, \lambda\right)\right], \\
R_{D_{3}} & =\left[\left(\lambda, \dagger_{3} / \lambda, \lambda\right)\right], \\
R_{D_{4}} & =\left[\left(\lambda, \dagger_{4} / \lambda, \lambda\right)\right] .
\end{aligned}
$$

A sample derivation is given as follows:

$$
\begin{aligned}
& \downarrow^{\downarrow} \dagger_{1} \dagger_{2}^{\downarrow} \dagger_{3} \dagger_{4} \Longrightarrow R_{I_{1}} \underline{a}^{\downarrow} \dagger_{1} \dagger_{2} \underline{u}^{\downarrow} \dagger_{3} \dagger_{4} \\
& \Longrightarrow R_{I_{1}} a \underline{u} \dagger_{1}^{\downarrow} \dagger_{2} u \underline{a}^{\downarrow} \dagger_{3}^{\downarrow} \dagger_{4} \\
& \Longrightarrow R_{I_{2}} a u \dagger_{1} \underline{g}^{\downarrow} \dagger_{2} u a^{\downarrow} \dagger_{3}^{\downarrow} \underline{c} \dagger_{4} \\
& \Longrightarrow R_{I_{2}} a u \dagger_{1} g \underline{u} \dagger_{2}^{\downarrow} u a \dagger_{3} \underline{a} c^{\downarrow} \dagger_{4} \\
& \Longrightarrow R_{I_{3}} a u \dagger_{1} g u \dagger_{2}^{\downarrow} \underline{u} u a \dagger_{3} a c \underline{a}^{\downarrow} \dagger_{4} \\
& \Longrightarrow R_{I_{3}} a u \dagger_{1}^{\downarrow} g u \dagger_{2} \underline{c u u a} \dagger_{3} \text { acag } \dagger_{4} \\
& \Longrightarrow_{R_{I_{4}}} \text { au } \dagger_{1}^{\downarrow} \underline{g} g u \dagger_{2} \text { cuua } \dagger_{3} \text { acag } \dagger_{4} \\
& \Longrightarrow R_{I_{4}} \text { au } \dagger_{1} \underline{c} g g u \dagger_{2} \text { cuua }_{{ }_{3}} \text { acag } \dagger_{4} \\
& \Longrightarrow_{R_{D_{1}}} a^{\Downarrow} \mathrm{cggu} \dagger_{2} \text { cuua }_{3} \text { acag } \dagger_{4} \\
& \Longrightarrow R_{D_{2}} \text { aucggu }{ }^{\Downarrow} \text { cuua }_{3} \text { acag } \dagger_{4} \\
& \Longrightarrow R_{D_{3}} \text { aucggucuua }{ }^{\Downarrow} \text { acag } \dagger_{4} \\
& \Longrightarrow R_{D_{4}} \text { aucggucuuaacag }{ }^{\Downarrow} \text {. }
\end{aligned}
$$

\section{Conclusion}

In this paper, we introduced the matrix insertion-deletion system and, using it, we modelled several bio-molecular structures that occur at the intramolecular, intermolecular and RNA secondary structure level. These structures are often discussed in the literature, thus they can be assumed to be important bio-molecular structures.

We remark that, in this paper, to model all the bio-molecular structures we used a matrix of insertion rules and a matrix of deletion rules separately (i.e., the system has no insertion rule and deletion rule together in a matrix) thus forming a new subclass. In all the systems we considered here, the insertion rule uses context and the deletion rule uses no context. This can be viewed as follows: the insertion operation works in a context-sensitive manner whereas the deletion operation works in a context-free manner. Thus, the system uses both the nature of context-sensitiveness and context-freeness, and it seems to be a promising model for application to various domains such as molecular biology and linguistics. With matrices consisting of both insertion and deletion rules, more complicated structures such as tertiary structures, quaternary structures, protein secondary structures (Mamitsuka and Abe, 1994) can be modelled, and this is left as a future research work.

From the computational linguistic point of view, one natural question is where to place this newly introduced formalism among the other formalisms that exist in this domain. Though the parsing algorithm for tree adjoining grammars $(T A G)$ and linear indexed grammar take $O\left(n^{6}\right)$ (Pardo et al., 1997), they have their own deficiency. For example, TAG cannot generate $L_{i}=$ $\left\{a_{1}^{n} a_{2}^{n} \ldots a_{i}^{n} \mid n \geq 1\right\}$ for $i \geq 5$ and the triple copy 
language $L_{3 c p}=\left\{w w w \mid w \in\{a, b\}^{*}\right\}$. However, multi-component TAG (MCTAG) can cover them. But there are languages like $L_{n s l}=\left\{a^{2^{n}} b^{n} \mid n \geq 1\right\}$ that cannot be covered by MCTAG, although, on the other hand, they are accepted by a Turing machine in polynomial time (Boullier and Sagot, 2011). With the introduced subclass variant, we can easily generate $L_{i}, i \geq 5$ and $L_{3 c p}$ languages; however, to generate $L_{n s l}$, it seems required that both insertion and deletion rules be used together in a matrix. We suppose that the introduced grammar formalism can emulate $T A G$, but subsumed by the range concatenation grammar $(R C G)$. Analyzing the relationship among these grammar formalisms with the introduced formalism in detail is out of objective of this paper, but can be carried out as a future work.

\section{Acknowledgment}

The first author would like to acknowledge the project SR/S3/EECE/054/2010, Department of Science and Technology, New Delhi, India. The second author is on leave from the parent institute at VIT University, Vellore, India. The authors would also like to thank the anonymous referees for their useful suggestions and corrections.

\section{References}

Boullier, P. and Sagot, B. (2011). Multi-component tree insertion grammars, in P. De Groote et al. (Eds.), Formal Grammar 2009, Lecture Notes in Artificial Intelligence, Vol. 5591, Springer, Berlin/Heidelberg, pp. 31-46.

Brendel, V. and Busse, H.G. (1984). Genome structure described by formal languages, Nucleic Acids Research 12(5): 2561-2568

Brown, M. and Wilson, C. (1995). RNA pseudoknot modelling using intersections of stochastic context free grammars with applications to database search, Proceedings of the Pacific Symposium on Biocomputing, Big Island, HI, USA, pp. 109-125.

Cai, L., Russell, L. and Wu, Y. (2003). Stochastic modelling of RNA pseudoknotted structures: A grammatical approach, Bioinformatics 19(1): 66-73.

Calude, C.S. and Paŭn, Gh. (2001). Computing with Cells and Atoms: An Introduction to Quantum, DNA and Membrane Computing, Taylor and Francis, London.

Chiang, D., Joshi, A.K. and Searls, D.B. (2006). Grammatical representations of macromolecular structure, Journal of Computational Biology 13(5): 1077-1100.

Dong, S. and Searls, D.B. (1994). Gene structure prediction by linguistic methods, Genomics 23(3): 540-551.

Dorigo, M. and Stutzle, T. (2004). Ant Colony Optimization, MIT Press, Cambridge, MA.

Durbin, R., Eddy, S., Krogh, A. and Mitchison, G. (1998). Biological Sequence Analysis, Cambridge University Press, Cambridge.
Eiben, A.E. and Smith, J.E. (2003). Introduction to Evolutionary Computing, Springer, Berlin/Heidelberg.

Galiukschov, B.S. (1981). Semicontextual grammars, Matematicheskaya Logika $i$ Matematicheskaya Lingvistika: 38-50, (in Russian).

Goldberg, E.D. (1989). Genetic Algorithms in Search, Optimization and Machine Learning, Addison-Wesley, Boston, MA.

Haussler, D. (1982). Insertion and Iterated Insertion as Operations on Formal Languages, Ph.D. thesis, University of Colorado, Boulder, CO.

Haussler, D. (1983). Insertion languages, Information Science 131(1): 77-89.

Head, T. (1987). Formal language theory and DNA: An analysis of the generative capacity of specific recombinant behaviors, Bulletin of Mathematical Biology 49(6): 737-750.

Kuppusamy, L., Mahendran, A. and Krishna, S.N. (2011a). Matrix insertion-deletion systems for bio-molecular structures, in R. Natarajan and A. Ojo (Eds.), ICDCIT2011, Lecture Notes in Computer Science, Vol. 6536, Springer, Berlin/Heidelberg, pp. 301-311.

Kuppusamy, L., Mahendran, A. and Clergerie, E.V. (2011b). Modelling intermolecular structures and defining ambiguity in gene sequences using matrix insertion-deletion systems in biology, computation and linguistics, in G.B. Enguix et al. (Eds.), New Interdisciplinary Paradigms, IOS Press, Amsterdam, pp. 71-85.

Lyngso, R.B., Zuker, M. and Pedersen, C.N.S. (1999). Internal loops in RNA secondary structure prediction, RECOMB99, Proceedings of the 3rd International Conference on Computational Molecular Biology, Lyon, France, pp. 260-267.

Lyngso, R.B. and Pedersen, C.N.S. (2000). Pseudoknots in RNA secondary structure, RECOMBO0, Proceedings of the 4th Annual International Conference on Computational Molecular Biology, Tokyo, Japan pp. 201-209.

Mamitsuka, H. and Abe, N. (1994). Prediction of beta-sheet structures using stochastic tree grammars, Proceedings of the 5th Workshop on Genome Informatics, Yokohama, Japan, pp. 19-28.

Pardo, M.A.A., Clergerie, E.V. and Ferro, M.V. (1997). Automata-based parsing in dynamic programming for LIG, in A.S. Narinyani (Ed.), Proceedings of the DIALOGUE'97 Computational Linguistics and Its Applications Workshop, Moscow, Russia, pp. 22-27.

Păun, Gh., Rozenberg, G. and Salomaa, A. (1998). DNA Computing: New Computing Paradigms, Springer, Berlin/Heidelberg.

Păun, Gh. (2002). Membrane Computing: An Introduction, Springer, Berlin/Heidelberg.

Petre, I. and Verlan, S. (2012). Matrix insertion-deletion systems, Theoretical Computer Science 456: 80-88.

Rivas, E. and Eddy, S.R. (2000). The language of RNA: A formal grammar that includes pseudoknots, Bioinformatics 16(4): 334-340. 
Rozenberg, G. and Salomaa, A. (1997). Handbook of Formal Languages, Vol. 1, Springer, New York, NY.

Sakakibara, Y., Brown, R., Hughey, R., Mian, I.S., Sjolander, K., Underwood, R.C. and Haussler, D. (1996). Stochastic context-free grammars for tRNA modelling, Nucleic Acids Research 22(23): 5112-5120.

Sakakibara, Y. (2003). Pair hidden Markov models on tree structures, Bioinformatics 19(1): 232-240.

Searls, D.B. (1988). Representing genetic information with formal grammars, Proceedings of the National Conference on Artificial Intelligence, Saint Paul, MN, USA, pp. 386-391.

Searls, D.B. (1992). The linguistics of DNA, American Scientist 80(6): 579-591.

Searls, D.B. (1993). The computational linguistics of biological sequences, in L. Hunter (Ed.), Artificial Intelligence and Molecular Biology, AAAI Press, Paolo Alto, CA, pp. 47-120.

Searls, D.B. (1995). Formal grammars for intermolecular structures, 1st International IEEE Symposium on Intelligence and Biological Systems, Washington, DC, USA, pp. 30-37.

Searls, D.B. (2002). The language of genes, Nature 420(6912): 211-217.

Theis, C., Janssen, S. and Giegerich, R. (2010). Prediction of RNA secondary structure including kissing hairpin motifs, Proceedings of WABI 2010, Liverpool, UK, pp. 52-64.

Uemura, Y, Hasegawa, A., Kobayashi, S. and Yokomori, T. (1999). Tree adjoining grammars for RNA structure prediction, Theoretical Computer Science 210(2): 277-303.

Yuki, S. and Kasami, T. (2006). RNA pseudoknotted structure prediction using stochastic multiple context-free grammar, IPSJ Transactions on Bioinformatics 47: 12-21.

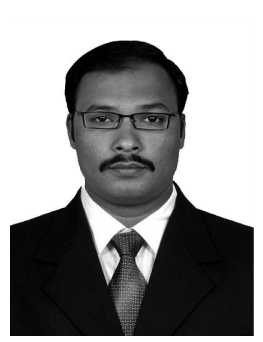

Lakshmanan Kuppusamy received his Ph.D from IIT Madras, India (2004), in theoretical computer science. His research interests include formal language theory and automata, bioinspired computing models and epistemic logic. He has published more than 30 papers in international journals and refereed international conferences. He is currently working as a professor in the School of Computing Science and Engineering at VIT University, Vellore, India.

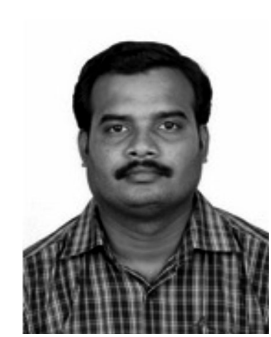

Anand Mahendran received his Ph.D. (computer science and engineering) from VIT University, India, in 2012, his M.E. (computer science and engineering) from Anna University, India, in 2005, and his B.E. (computer science and engineering) from VIT University, India, in 2003. His research interests include formal language theory and automata, and bio-inspired computing models. He has published more than 20 papers in international journals and refereed international conferences. He works as an assistant professor in the College of Computer Science and Information Systems, Jazan University, Kingdom of Saudi Arabia.

Received: 8 August 2014

Revised: 17 March 2015

Re-revised: 30 July 2015 